\title{
Cognitive Radio Techniques under Practical Imperfections: A Survey
}

\author{
Shree Krishna Sharma, Member, IEEE, Tadilo Endeshaw Bogale, Member, IEEE, \\ Symeon Chatzinotas, Senior Member, IEEE, Björn Ottersten, Fellow, IEEE, \\ Long Bao Le, Senior Member, IEEE, Xianbin Wang, Senior Member, IEEE
}

\begin{abstract}
Cognitive Radio (CR) has been considered as a potential candidate for addressing the spectrum scarcity problem of future wireless networks. Since its conception, several researchers, academic institutions, industries, regulatory and standardization bodies have put their significant efforts towards the realization of CR technology. However, as this technology adapts its transmission based on the surrounding radio environment, several practical issues may need to be considered. In practice, several imperfections such as noise uncertainty, channel/interference uncertainty, transceiver hardware imperfections, signal uncertainty, synchronization issues, etc. may severely deteriorate the performance of a CR system. To this end, the investigation of realistic solutions towards combating various practical imperfections is very important for successful implementation of the cognitive technology. In this direction, first, this survey paper provides an overview of the enabling techniques for CR communications. Subsequently, it discusses the main imperfections that may occur in the most widely used CR paradigms and then reviews the existing approaches towards addressing these imperfections. Finally, it provides some interesting open research issues.
\end{abstract}

Index Terms - Cognitive radio, channel uncertainty, noise uncertainty, spectrum sensing, transceiver imperfections, underlay

\section{INTRODUCTION}

The demand for broadband wireless spectrum is rapidly increasing while its supply is limited due to spectrum segmentation and the dedicated frequency allocation of standardized wireless systems. This scarcity has led to the concept of Cognitive Radio (CR) communication which comprises a variety of techniques enabling the coexistence of licensed and unlicensed systems over the same spectrum. Recent technical advances in Software Defined Radio (SDR), wideband transceivers, digital signal processors, etc., have made it possible to employ intelligent transceivers, hence leading to the possibility of utilizing the available spectrum in a very dynamic and adaptive manner.

Copyright (c) 2015 IEEE. Personal use of this material is permitted. However, permission to use this material for any other purposes must be obtained from the IEEE by sending a request to pubs-permissions@ieee.org.

This work was partially supported by the National Research Fund, Luxembourg under the CORE projects "SeMIGod" and "SATSENT", the Natural Sciences and Engineering Research Council of Canada (NSERC) under the project CRDPJ $461894-13$, and Nutaq Inc.

S. K. Sharma, S. Chatzinotas and B. Ottersten are with the SnT (http://www.securityandtrust.lu), University of Luxembourg, L-2721, Luxembourg, Email: \{shree.sharma, symeon.chatzinotas, bjorn.ottersten\}@uni.lu.

T. E. Bogale and Long B. Le are with the INRS, Université du Québec, Montréal, QC, Canada, and X. Wang is with the University of Western Ontario, London, ON, Canada. Email: \{tadilo.bogale, long.le\}@emt.inrs.ca and \{xianbin.wang\}@uwo.ca.
Although the CR paradigm started with the idea of an SDR [1], it may comprise a wide variety of techniques and architectures in order to allow the spectral coexistence of the licensed and unlicensed wireless systems. It has a wide range of application areas ranging from Television WhiteSpaces (TVWSs) [2] to satellite communications [3], [4] and several industry players are putting significant efforts towards the realisation of CR technology [5]. In CR terminology, Primary (licensed) Users (PUs), also called incumbent users, can be defined as the users who have higher priority or legacy rights on the use of a specific part of the spectrum. On the other hand, Secondary (unlicensed) Users (SUs), also called cognitive users, exploit this spectrum in such a way that they do not provide harmful interference to the normal operation of the licensed PUs. The SU needs to have CR capabilities in order to acquire information about its operating environments and to adapt its radio parameters autonomously in order to exploit the underutilized part of the spectrum.

Since the conception of the term "CR" by J. Mitola in the late 1990's [1], several researchers and industrial/academic/regulatory bodies have attempted to provide different definitions of the $\mathrm{CR}$ as well as different forms of cognitive cycles [1], [6], [7]. In this context, there are two widely used approaches for enhancing the spectral efficiency of current wireless systems: (i) by utilizing opportunistic spectrum access, called Dynamic Spectrum Access (DSA), and (ii) by allowing the sharing of the available spectrum between primary and secondary systems, called spectrum sharing. Further, the most common and basic functions available in all the proposed cognitive cycles are: (i) Spectrum awareness, (ii) Analysis and decision, and (iii) Spectrum exploitation (adaptation). These functions need to be carried out repeatedly until full adaptation to the changed environmental condition is achieved. Any other CR functionalities can be considered to be just the variants of these basic tasks.

While considering the aforementioned simplified cognitive cycle, the first task for a CR is to be aware of its surrounding radio environment, i.e., spectrum awareness. The next step is to analyze the acquired information and to take an intelligent decision on how to use the available resources effectively. Subsequently, the CR autonomously adapts its operational parameters such as transmit power, operating frequency, modulation and coding scheme, etc. to any environmental conditions in order to exploit the available spectral opportunities effectively, called spectrum exploitation. More specifically, spectrum awareness capability helps a CR to acquire information 


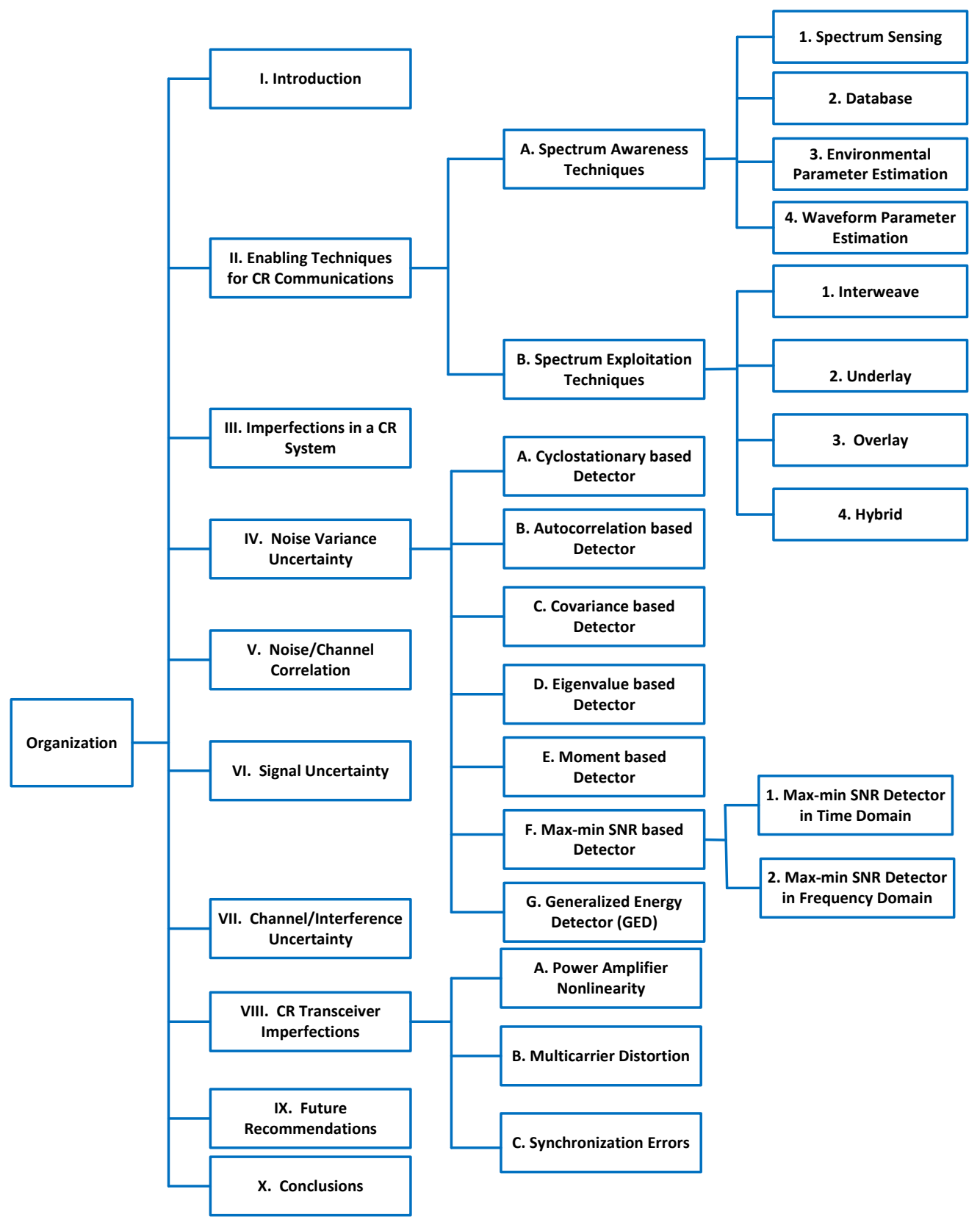

Fig. 1. Structure of the paper

about the spectral opportunities dynamically while spectrum exploitation capability assists a CR to exploit the available spectral opportunities efficiently.

Several survey papers exist in the literature in the context of CR communications covering a wide range of areas such as spectrum occupancy measurement campaigns [8], Spectrum Sensing (SS) [9]-[13], spectrum management [14], emerging applications [15], spectrum decision [16], spectrum assignment [17], spectrum access strategies [18], resource allocation [19], [20], machine-learning techniques [21] and CR networks [22]. However, to the best of authors' knowledge, no contributions have been reported in the direction of providing a comprehensive review of the existing CR approaches considering practical imperfections. Despite the sig- nificant volume of available literature under ideal conditions, investigation of realistic solutions towards combating various practical imperfections has become critical towards its actual deployment. Existing contributions have either neglected the practical constraints or are applicable to a specific scenario under a single practical imperfection. In practice, a number of practical imperfections should be considered by a designer while implementing a CR system.

The main imperfections that may occur in practical scenarios are noise uncertainty, channel/interference uncertainty, CR transceiver imperfections, noise/channel correlation, signal uncertainty, etc. as detailed later in Section III. In this context, it remains an open challenge to study the effect of different practical imperfections on the performance of a CR transceiver. 
TABLE I

DEFINITIONS OF ACRONYMS AND NOTATIONS

\begin{tabular}{|c|c|c|c|}
\hline Acronyms/Notations & Definitions & Acronyms/Notations & Definitions \\
\hline $\mathrm{ACl}$ & Adjacent Channel Interference & PU & Primary User \\
\hline ADC & Analog to Digital Converter & QAM & Quadrature Amplitude Modulation \\
\hline $\mathrm{AGC}$ & Automatic Gain Controller & QoS & Quality of Service \\
\hline AWGN & Additive White Gaussian Noise & QPSSK & Quadrature Phase-Shift Keying \\
\hline BEP & Bit Error Probability & REM & Radio Environment Map \\
\hline BER & Bit Error Rate & RF & Radio Frequency \\
\hline BPSK & Binary Phase Shift Keying & ROC & Receiver Operating Characteristic \\
\hline $\mathrm{CAC}$ & Cyclic Autocorrelation & RMT & Random Matrix Theory \\
\hline CDR & Constant Detection Rate & $\mathrm{SCN}$ & Signal Condition Number \\
\hline CDMA & Code Division Multiple Access & SDR & Software Defined Radio \\
\hline CED & Conventional Energy Detection & SISO & Single Input Single Output \\
\hline CFAR & Constant False Alarm Rate & SIMO & Single Input Multiple Output \\
\hline $\mathrm{CP}$ & Cyclic Prefix & SLE & Scaled Largest Eigenvalue \\
\hline $\mathrm{CPC}$ & Cognitive Pilot Channel & SS & Spectrum Sensing \\
\hline CR & Cognitive Radio & ST & Secondary Transmitter \\
\hline $\mathrm{CS}$ & Compressive Sensing & SR & Secondary Receiver \\
\hline CSI & Channel State Information & SINR & Signal to Interference plus Noise Ratio \\
\hline DoA & Direction of Arrival & SNR & Signal to Noise Ratio \\
\hline DSA & Dynamic Spectrum Access & SRRCF & Square Root Raised Cosine Filter \\
\hline DA & Data-Aided & TDD & Time Division Duplex \\
\hline DVB & Digital Video Broadcasting & TVWS & Television WhiteSpace \\
\hline ED & Energy Detection & SU & Secondary User \\
\hline EIRP & Effective Isotropic Radiated Power & WRAN & Wireless Regional Area Network \\
\hline EME & Energy to Minimum Eigenvalue & USRP & Universal Software Radio Peripheral \\
\hline EVD & Eigenvalue Decomposition & $\operatorname{diag}(\cdot)$ & Diagonalization \\
\hline EZ & Exclusion Zone & $E[\cdot]$ & Expectation \\
\hline FCC & Federal Communications Commission & $H_{0}$ & Noise only hypothesis \\
\hline FDD & Frequency Division Duplex & $H_{1}$ & Signal plus noise hypothesis \\
\hline FC & Fusion Centre & $\rho$ & Correlation coefficient \\
\hline GED & Generalized Energy Detector & $\operatorname{erfc}(\cdot)$ & Complementary error function \\
\hline GLRT & Generalized Likelihood Ratio Test & $P_{d}$ & Probability of detection \\
\hline HNP & Hidden Node Problem & $P_{f}^{a}$ & Probability of false alarm \\
\hline HPA & High Power Amplifier & $\sigma^{2}$ & Noise variance \\
\hline i.i.d. & independent and identically distributed & $\gamma$ & SNR \\
\hline JD & John's Detector & $\alpha$ & Cyclic frequency \\
\hline LO & Local Oscillator & $\tau$ & Time lag \\
\hline MIMO & Multiple Input Multiple Output & $B$ & Bandwidth \\
\hline ModCod & Modulation and Coding & $\beta$ & Excess bandwidth \\
\hline MCS & Modulation and Coding Scheme & $N$ & Number of samples \\
\hline ME & Maximum Eigenvalue & $M$ & Number of receive dimensions \\
\hline MME & Maximum to Minimum Eigenvalue & $L$ & Oversampling factor \\
\hline NDA & Non-Data-Aided & $\sum$ & Summation \\
\hline NC-OFDM & Non-Contiguous OFDM & $\hat{\mathbf{R}}_{\mathbf{X}}$ & Sample covariance of $\mathbf{X}$ \\
\hline OFDM & Orthogonal Frequency Division Multiplexing & $I_{T}$ & Interference threshold \\
\hline OSI & Open System Interconnection & $h$ & Channel fading coefficient \\
\hline PA & Power Ámplifier & $T$ & Test statistic \\
\hline PAPR & Peak to Average Power Ratio & $\lambda$ & Sensing threshold \\
\hline PT & Primary Transmitter & $\mathcal{C}$ & Complex numbers \\
\hline PR & Primary Receiver & $(\cdot)^{\dagger}$ & Conjugate transpose \\
\hline
\end{tabular}

In this direction, the main focus of this survey paper is to provide a comprehensive review of $\mathrm{CR}$ techniques under practical imperfections. First, we provide a brief overview of the existing CR approaches considering spectrum awareness and exploitation techniques. Subsequently, we highlight the major imperfections that may occur in practice considering the main CR paradigms widely considered in the literature. Then, we review the related existing techniques which take these imperfections into account. Finally, we provide interesting open research issues.

The remainder of this paper is structured as follows: Section II provides an overview of the existing enabling techniques for CR communications. Section III highlights the main practical imperfections that may arise in a practical $\mathrm{CR}$ system. Then Section IV focuses on noise uncertainty problem including its modeling and possible solutions, and describes all the existing approaches under noise variance uncertainty. Section $\mathrm{V}$ reviews the existing works under noise/channel correlation while Section VI discusses the signal uncertainty problem and reviews related works. Section VII identifies the causes and the effects of channel/interference uncertainty referring to the current state of the art. Subsequently, Section VIII discusses various imperfections in a CR transceiver which may result from hardware components of an RF chain. Finally, Section IX provides open research issues and Section $\mathrm{X}$ concludes this paper. In order to improve the flow of this paper, we provide the structure of the paper in Fig. 1 and the definitions of acronyms/notations in Table I.

\section{EnAbling Techniques for CR Communications}

As highlighted in Section I, the main capabilities required for a CR are spectrum awareness, spectrum decision and spectrum exploitation. These functionalities can be implemented in different layers of a widely used Open System Interconnection (OSI) protocol stack. While relating a CR architecture with the OSI model, the following mapping can be made between OSI layers and cognitive capabilities [23]: (i) context awareness for higher layer (application), (ii) inter-operability for intermediate layers (transport and network), and (iii) link adaptation for lower layers (physical and data link). The focus of this survey paper will be on the lower layers of this protocol stack, mostly from physical layer perspective.

The two main functions of a $\mathrm{CR}$ are to acquire information about spectral opportunities by monitoring the surrounding radio environments and to exploit those opportunities in an intelligent way while guaranteeing the normal operation of the 


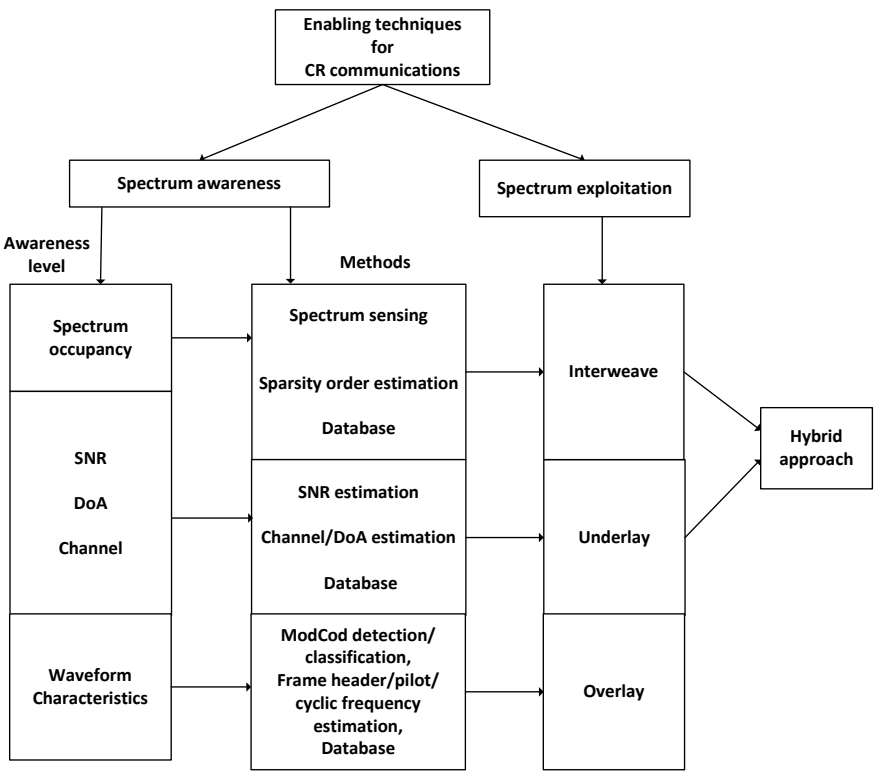

Fig. 2. Mapping of spectrum awareness techniques with spectrum exploitation techniques

licensed PUs. In this context, we broadly categorize a wide range of $\mathrm{CR}$ enabling techniques available in the literature into: (i) spectrum awareness techniques, and (ii) spectrum exploitation techniques. In Fig. 2, we provide the mapping of spectrum awareness mechanisms with different spectrum exploitation methods [24]. Subsequently, we provide the classification of the existing approaches and a brief description of these approaches in the following subsections.

\section{A. Spectrum Awareness Techniques}

Depending on the employed techniques at the CR node, different levels of awareness can be achieved as illustrated in Fig. 2. The acquired information can be spectrum occupancy over the available bands, Signal to Noise Ratio (SNR) of the PU signal, channel towards the PUs, modulation and coding used by the PUs, pilot/header information in the PU transmit frame, etc. From the spectrum utilization point of view, the greater the awareness level at the CR node, the better becomes the spectrum utilization. For acquiring information about the spectrum occupancy, SS [9]-[12], database [25][31] and beacon transmission-based [32] methods can be applied. Further, for acquiring information about PU SNR and channels, suitable SNR and channel estimation algorithms can be exploited. In addition, the waveform characteristics of the PU signals can be useful for realizing overlay communication. The waveform characteristics are the specific features of the PU signals and may include information about the Modulation and Coding (ModCod) scheme used by the PUs [33], [34], cyclic frequencies, pilot/header information used in PU transmission, etc. For acquiring these features, suitable ModCod detection/classification, frame header/pilot estimation approaches can be utilized.

In Fig. 3, we present various spectrum awareness techniques. Based on whether the primary system's parameters are required or not during the spectrum awareness process, spectrum awareness techniques can be broadly categorized into: (i) blind and (ii) non-blind. Blind ${ }^{1}$ spectrum awareness techniques do not require information about the parameters of the primary system whereas, non-blind spectrum awareness techniques require specific information about the parameters of the primary system. As noted in Fig. 3, blind spectrum awareness methods can be categorized into: (i) blind SS, and (ii) environmental parameter estimation. Similarly, nonblind spectrum awareness methods can be categorized into: (i) non-blind SS, (ii) database, and (iii) waveform parameter estimation methods. These approaches are briefly discussed below.

1. Spectrum Sensing (SS): SS is an important mechanism in order to acquire the spectrum occupancy information of the primary spectrum. In other words, it exploits the spectral holes in several domains such as time, frequency, space, polarization, and angular domains. The ST is assumed to be equipped with a Radio Frequency (RF) chain having sensing capability and senses the presence or absence of the PUs with the help of the employed signal processing technique. Based on the employed signal processing technique, the main SS techniques discussed in the literature are Energy Detection $(E D)^{2}$, feature detection, matched filter based detection, autocorrelation based detection, covariance based detection, eigenvalue based detection, etc.

As depicted in Fig. 3, some of these techniques are blind in terms of the requirement of the primary system's parameters whereas, others are non-blind. Further, many of these techniques require the knowledge of noise covariance at the $\mathrm{CR}$ receiver. Mostly eigenvalue based approaches [35], [36] and some of the recently proposed feature detectors [37]-[39] are blind in terms of primary system's parameters, channel as well as the noise variance. In Fig. 4, we present the classification of SS techniques on different bases [24]. Interested readers may refer to [9]-[12] for the detailed explanation and the related references for various SS techniques.

In practical wireless fading channels, the SS efficiency of the aforementioned techniques may be degraded due to Hidden Node Problem (HNP), path loss, shadowing, multipath fading and receiver noise/interference uncertainty issues. The HNP arises when a cognitive user experiences a deep fade or shadowing from the Primary Transmitter (PT) and cannot detect the presence of the primary signal, leading to the missed detection, and hence the harmful interference to the Primary Receiver (PR). In this context, cooperative SS, in which several nodes cooperate with each other in order to enhance the overall sensing performance, has been considered as a promising approach [12], [40], [41]. The main concept behind cooperative SS is to enhance the sensing performance by exploiting the observations captured by spatially located CR users as depicted in Fig. 5.

The cooperation can be among the CR nodes or external

${ }^{1}$ It should be noted that the definition of blindness here is independently of the noise covariance knowledge at the $\mathrm{CR}$ and is entirely dependent on whether the knowledge about the primary system's parameters is required or not.

${ }^{2}$ This is also referred as Conventional Energy Detection (CED) later in Section IV. 


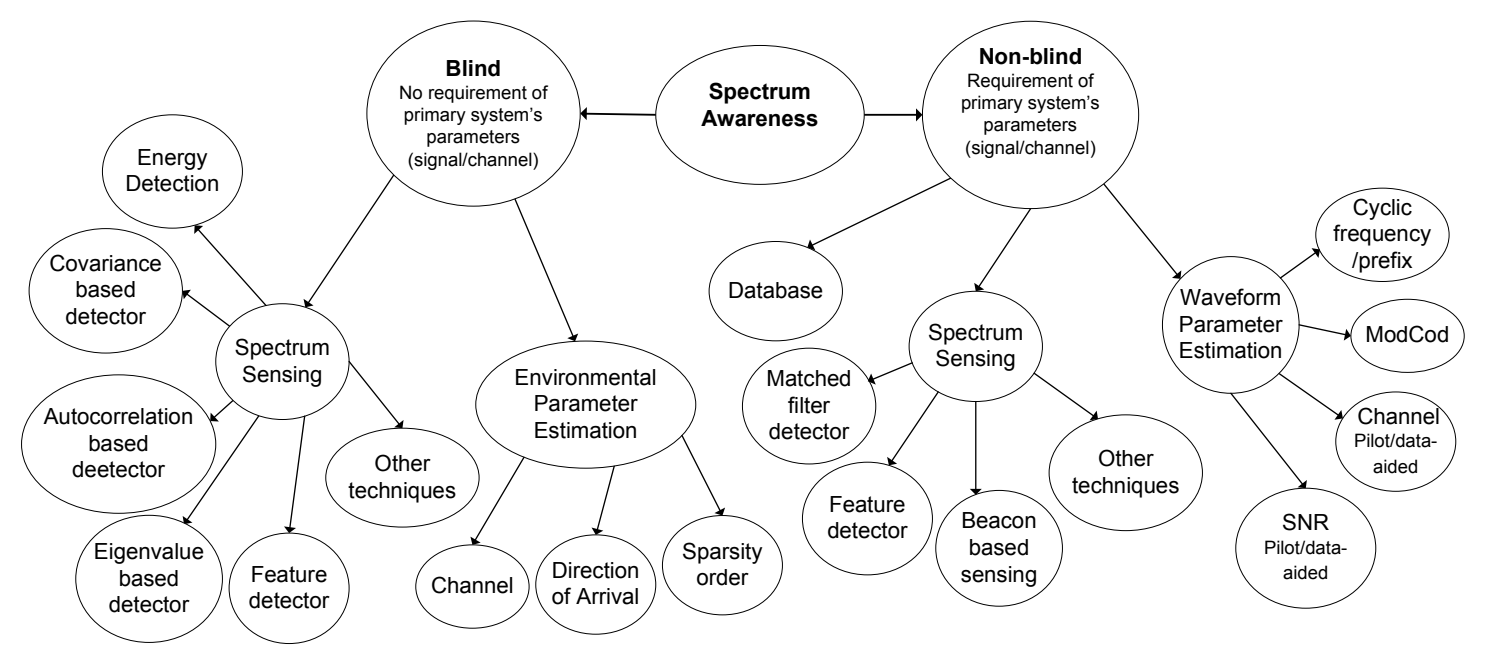

Fig. 3. Classification of spectrum awareness techniques

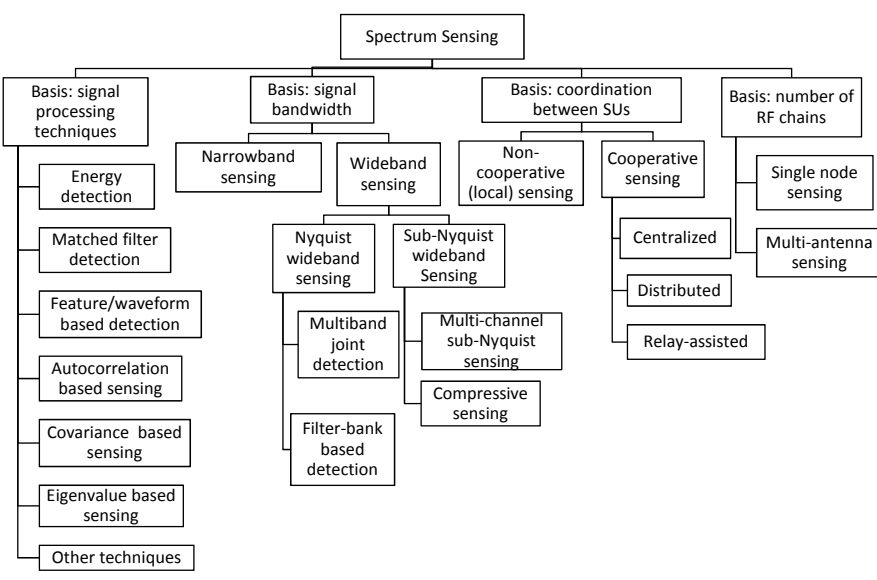

Fig. 4. Classification of SS techniques

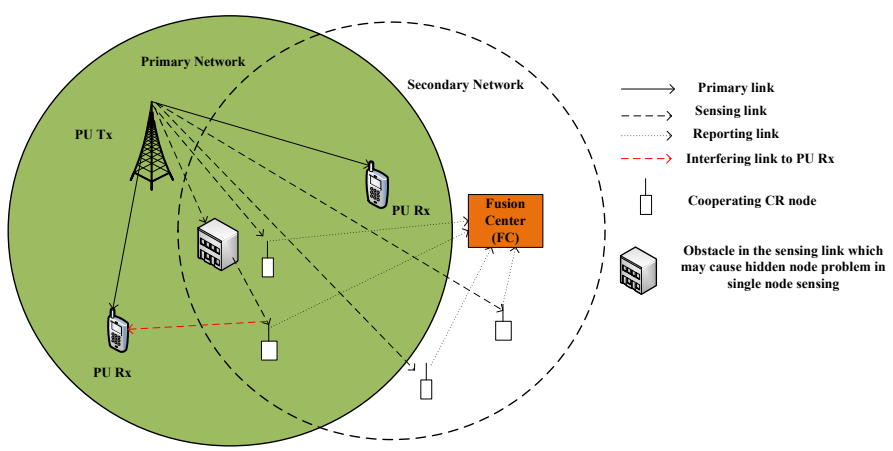

Fig. 5. Schematic of the cooperative SS scenario

sensors can be deployed to build a cooperative sensing network. In the former case, the cooperation can be implemented in the following ways [40]: (i) centralized, (ii) distributed, and (iii) relay-assisted. In the centralized approach, a central unit collects sensing information from cognitive devices, identifies the available spectrum, and broadcasts this information to other CRs or directly controls the CR traffic whilst in the distributed approach, cognitive nodes share information among each other but they make their own decisions regarding which part of the spectrum they can use. Distributed sensing is more advantageous than the centralized sensing since it does not require a backbone infrastructure and it has reduced cost. In the relay-assisted approach, a CR user with a strong sensing channel and a weak report channel can cooperate with a CR user observing a weak sensing channel and a strong report channel in order to improve the overall sensing performance. Despite several advantages of cooperative approach such as cooperative gain, relaxed sensitivity [12], the cooperation burden, which arises due to additional operations devoted to cooperative sensing, can be a critical issue from a practical perspective. The detailed survey on existing cooperative SS approaches can be found in [12], [40].

Moreover, based on the bandwidth of the channel to be sensed, SS techniques can be narrowband and wideband. Most of the aforementioned techniques are narrowband and can be extended for wideband context using complex hardware circuitry. Existing wideband sensing techniques include tunable filter method [42], Wavelet transform method [43], Multibeam joint detection [44], Spatial-spectral joint detection [45], and Compressive Sensing (CS)-based detection [42], [46]. Further, collaborative wideband sensing has been discussed in the several literature for enhancing the detection performance in the presence of fading [45], [47]. The collaboration among multiple spatially distributed CRs can relax the sensitivity constraint on the RF front-end by enhancing the signal detection at the fusion center and may even broaden the frequency range of SS.

The CS technique performs sampling at a rate less than the Nyquist rate and reconstructs the original signal back from significantly lower number of compressive measurements. In addition, different signal processing operations such as detection, estimation and filtering can be carried out directly from compressive measurements without the requirement of full signal recovery, hence reducing the sensing hardware cost significantly [48]. Several hardware architectures have been proposed and implemented in the literature enabling the compressive samples to be acquired in practical settings. Some of the examples are [11]: (i) Random Filtering, (ii) Com- 
pressive multiplexer, (iii) Random convolution, (iv) Random demodulator, and (v) Modulated wideband converter.

As noted in Fig. 3, one of the non-blind spectrum awareness approaches is beacon-based SS method in which the beacon signals are broadcasted in appropriate signalling channels by the primary systems and CR users detect these signals in order to obtain information about the spectrum occupancy. The beacon signals may carry information about the spectrum usage of the primary system, traffic trends, and future frequency usage, etc. [32] and also the information from the database in database-assisted systems. This approach requires an agreement between secondary and primary system operators in order to share the real time spectrum usage information of the primary network with the secondary network. The above interaction may require additional modifications in the primary legacy radio systems, which might be difficult to implement in practice. In this context, Cognitive Pilot Channel (CPC) has received important attention as a signalling channel in the scenarios of primary-secondary coexistence [49], [50].

The SS problem is basically to distinguish the cases of the PU presence and the PU absence. Denoting the hypotheses of the PU absence and the PU presence by $H_{0}$ (noise only hypothesis) and $H_{1}$ (signal plus noise hypothesis) respectively, this problem can be formulated in terms of a well known binary hypothesis testing problem in the following way [51]

$$
\begin{aligned}
y[n] & =z[n], & H_{0} \\
y[n] & =h s[n]+z[n], & H_{1}
\end{aligned}
$$

where $s[n]$ denotes the transmitted signal at the $n$th sampling instant, $h$ indicates the channel coefficient, $z[n]$ denotes the Additive White Gaussian Noise (AWGN) and $y[n]$ denotes the received signal at the $\mathrm{CR}$ at the $n$th sampling instant.

In order to test the above hypothesis, we need to find a decision statistic whose distribution sufficiently differs under the $H_{0}$ and the $H_{1}$ hypotheses. For example, the decision statistics for the ED approach is the received energy and the maximum eigenvalue for maximum eigenvalue based detection. The sensing threshold is usually calculated from the distribution of the decision statistics, i.e., received energy for the ED, under the $H_{0}$ hypothesis. If the hypothesis $H_{0}$ is satisfied, then it can be decided that the PU signal over a certain band is absent and then the SU can utilize this band on the secondary basis. Whereas, if the hypothesis $H_{1}$ is satisfied, the decision is the presence of the PU signal and secondary transmission should be switched to another band. In addition, if other bands in the available bandwidth range are not available or their quality is not satisfactory, the secondary transmission should be moved to the exclusive band. These decisions are to be taken centrally by the network management system based on the feedback it receives by the secondary terminals [52].

2. Database: Geolocation database is an alternative spectrum awareness approach to obtain the knowledge of the radio environment and can be used to query various levels of information required by the CRs. In this scheme, spectrum usage parameters of the primary system such as the place, time, frequency, etc. are stored in a centralized database. The
SUs who want to reuse the primary spectrum have to make a request to the database system and based on the availability of the unoccupied channels in that location and for the requested period, the database system can grant access to those channels.

A CR can also use the database approach including history information and prediction methods to make the operation more efficient. This approach is based on maintaining a frequently updated and centrally located database with information about the regional spectrum usage, locations of PTs/PRs, coverage areas, frequencies of operation, transmission powers, radio technologies, etc. This scheme is quite static and the dynamicity of this scheme depends on how fast the primary spectrum usage information is updated in the database. Several contributions in the literature have exploited this approach using the concept of a Radio Environment Map (REM) [25][31].

3. Environmental Parameter Estimation: In addition to spectrum occupancy information captured by the SS mechanism in a CR, several other parameters can be useful in designing the best transmission strategy for a CR. In other words, this is the additional level of awareness that a CR needs to have for controlling its power/radiation in order to protect the cochannel PUs. By environmental parameter estimation, we mean the estimation of the parameters related to the RF environment and not to the primary signal. As reflected in Fig. 3, the following parameters can be estimated from the surrounding environment blindly using blind estimation approaches: (i) channel, (iv) SNR, (ii) DoA and (iii) sparsity order. If the CR node can acquire information about SNR and channel of the primary signals, different underlay techniques such as Exclusion Zone (EZ), and power control [53] can be employed. Based on the estimated SNR, the CR can control its power in order to enhance its throughput while protecting the PUs at the same time. An accurate estimation of SNR further helps in link adaptation and iterative decoding.

The existing SNR estimation algorithms can be broadly categorized into [54]: (i) Data-Aided (DA), and (ii) Non-DataAided (NDA). The NDA estimators fall under this spectrum awareness category and they derive the SNR estimates directly from the unknown, information-bearing portion of the received signal. For SNR estimation problem in wireless communications literature, several techniques have been reported (see [54], [55] and references there in). However, in the context of CR networks, there exist only a few SNR estimation related works [36], [56]-[58]. Moreover, if the CR node has information on the DoA of the primary terminals either through database or through some DoA estimation algorithms, it can employ suitable beamforming based underlay approaches in order to mitigate interference towards/from the PRs/PTs [59], [60].

Sparsity order is another useful parameter in the context of a wideband CR and can be defined as the ratio of the occupied carriers to the total carriers over the band of interest. This parameter actually provides information about what percentage of the licensed band is available for the secondary usage. This awareness is helpful in implementing compressive sensingbased wideband sensing. Since the sparsity order is a time varying parameter and is not known to the CR receiver before- 

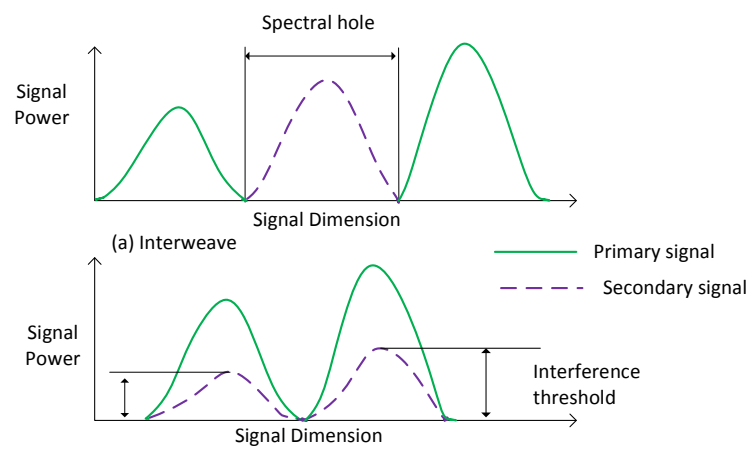

(b) Underlay

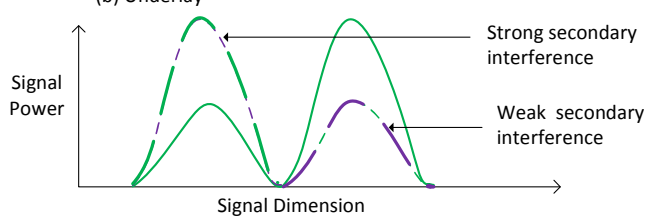

(c) Overlay

Fig. 6. Illustrations of interweave, underlay and overlay techniques

hand, it needs to be estimated in practice. If the information about the sparsity order is available to the wideband CR transceiver, it can dynamically adapt its sampling rate in order to fully exploit the advantages of the CS technique. In this context, estimating the sparsity order is crucial and has been studied in some existing contributions [61], [63].

4. Waveform Parameter Estimation: This awareness category includes parameter estimation methods that require side knowledge about the waveform used by the primary. The following awareness methods may fall under this category: (i) pilot-based/data-aided SNR/channel estimation [49], [64], [65], (ii) Cyclic frequency/prefix estimation [66]-[69], and (iii) ModCod detection and classification based on Modulation and Coding Scheme (MCS) [33], [34]. The data-aided $\mathrm{SNR} /$ channel estimators predict the SNR/channel values using the known data, for example, pilot sequences provided for synchronization and equalization [54].

\section{B. Spectrum Exploitation Techniques}

Based on the access technology of the SUs, the existing spectrum exploitation techniques can be broadly categorized into interweave, underlay, and overlay techniques [7]. Although different researchers have diverse opinions on the use of terminologies "interweave", "underlay" and "overlay" [7], [70], we follow the classification provided by A. Goldsmith, et al. in [7] and provide their illustrations in Fig. 6. Furthermore, we use the term "Hybrid" as in [71], [72] in order to denote the method which combines both interweave and underlay approaches. These methods are briefly discussed in the following paragraphs and interested readers may refer to [7] for the detailed explanation of interweave, underlay and overlay approaches and to [71], [72] for the hybrid approach. 1. Interweave: This paradigm encompasses interference avoidance or opportunistic techniques which require SUs to communicate opportunistically using spectral holes in space, frequency, and time which are not occupied by the PUs. Therefore, there occurs no interference in the ideal case [7]. This technique does not impose any restrictions on the transmit power of the SUs but is limited by the range of available spectral holes. Although space, time and frequency are common dimensions used for interweave communication, polarization [73], [74] and angular [75] domains can also be considered as additional dimensions for spectrum exploitation purpose. As depicted in Fig. 2, spectrum awareness techniques such as SS, database and sparsity order estimation facilitate the interweave communication.

2. Underlay: Underlay communication is characterized by strict constraints on the interference that may be caused by the STs to the PRs. In this paradigm, the coexistence of primary and secondary systems is allowed, only if the interference caused by STs to the PRs can be controlled and managed properly [76]. The maximum allowable interference level at the PR can be modeled by the concept of interference temperature, as defined by the Federal Communications Commission (FCC) spectrum policy task force in [77]. This metric is also termed as interference threshold which guarantees the reliable operation of the PUs regardless of the SU's spectrum utilization [78].

The STs utilizing the shared band must guarantee that their transmissions added to the existing interference must not exceed the interference threshold at the PR. As depicted in Fig. 2 , side information about primary system such as SNR, channels, DoAs are useful for realizing the underlay techniques. Furthermore, underlay communication can be realized with the help of one or many of the following methods: (i) cognitive beamforming with the help of multiple antennas [59], [60], (iii) cognitive resource allocation (carrier/power) at the ST, (ii) spread spectrum approaches by spreading the secondary signal below the noise floor and then de-spreading at the CR, (iv) cognitive interference alignment [79], and (v) exclusion zone.

3. Overlay: Overlay communication is characterized by the mitigation of interference with the help of advanced coding and transmission strategies at the STs. In this paradigm, the SU can transmit simultaneously with the PU and the interference caused by the ST to the PR can be offset by using a part of the SU's power to relay the primary message [7]. In this scheme, the SU transmits the PU signal along with its own signal. A two user interference channel where the ST has knowledge of the PU's message can be considered as a simplistic example of an overlay CR network. The cognitive transmitter should have knowledge of the PU's channel gains, codebooks and possibly its messages as well. Different precoding techniques such as Gel'fand-Pinsker binning and dirty paper coding in Gaussian channels can be applied to overlay networks [80] [81].

In practice, this paradigm is difficult to implement due to a high level of cognition required between primary and secondary systems. In this context, one possible approach is to acquire the waveform characteristics of the PU signal by using estimation approaches such as ModCod classification/detection, frame header/pilot/cyclic frequencies estimation, etc. as depicted in Fig. 2 and use these waveform characteristics in order to design advanced transmission strategies at the ST.

4. Hybrid: The SS approach ignores the interference toler- 
ance capability of the PUs focusing only on bursty PU traffic whereas, the possibility of having secondary transmission with full power is neglected in the underlay based approach [72]. More specifically, the underlay approach is not able to detect the activity or inactivity of the PUs in a particular band and hence does not utilize the idle bands efficiently. On the other hand, the SS approach does not allow the SUs to transmit in a particular frequency band when the PU is active in that band. To address this, a hybrid approach which can overcome the aforementioned drawbacks of both approaches has received important attention in the recent literature [71], [72], [82]. In this approach, a CR can access the PU channel with the full power in case of an idle channel and also can access the channel with the controlled power in case of the occupied channel. Some aspects of this approach which are recently dealt in the literature are: (i) transmit mode selection/switching [72], [82] and (ii) sensing-throughput tradeoff [71].

\section{IMPERFECTIONS IN A CR SYSTEM}

The spectrum awareness problem for a CR is different from the legacy wireless communication systems in the following ways [83]: (i) no prior knowledge of the PU signal structure, channel and noise variance, (ii) the information such as spectrum occupancy, SNR, etc. should be acquired at the shortest possible time, (iii) primary SNR may be very low due to fading and multi-path phenomenon, and (iv) noise/interference power varies with time in practical scenarios giving rise to noise/interference power uncertainty. The main imperfections which may degrade the performance of a CR system are listed below.

- Noise uncertainty: Noise is an unwanted random process which may arise from several sources in the external environment as well as from every components of a receiver chain. In the real-world scenarios, like other parameters, noise distribution is not known to infinite precision and the noise is neither perfectly Gaussian, perfectly white, nor perfectly stationary [84]. Therefore, the noise variance in practice has to be estimated by using a proper noise calibration method. The noise calibration can be done either during the manufacturing process or by carrying out on-site Out of Bands (OoB) measurements. Another option for noise calibration is to use in-band measurements at the frequencies where the pilot is absent so that the noise statistics can be calibrated at the pilot frequencies [85].

The noise estimation can be perfect in the ideal case, however, in practice, accurate estimation of the noise variance is not possible, thus limiting the performance of the noise statistics based sensing techniques e.g., the ED, at low SNRs. In the ED, the noise variance uncertainty may lead to the SNR wall phenomenon due to which it is not possible to achieve the robust detection performance beyond a certain SNR value even by increasing the sensing duration [84]. The noise uncertainty can be categorized into the following two types [83]: (a) receiver device noise uncertainty caused by components in the RF chain, and (b) environmental noise uncertainty caused by the surrounding environment.
- Channel/interference uncertainty: The channel information towards the PRs is critical for a CR in order to control the interference caused by it. In any case, the operation of the licensed primary system should not be degraded below an acceptable limit and an underlay $\mathrm{CR}$ has to operate under this constraint. In practice, channel information can be acquired using different channel estimation methods and this estimation may not be perfect due to channel uncertainty caused by time varying nature of the wireless channel as well as several other factors stated later in Section VII. Furthermore, the primary received power is never known accurately due to the presence of channel uncertainty, and thus needs to be estimated at the CR [86]. This received power estimation may not be perfect in practice, leading to the violation of the interference threshold constraints at the PRs. Furthermore, the interference may exist anywhere and anytime in the environment and its properties such as power level, waveform and distribution are usually unknown, leading to the problem of interference uncertainty in a practical CR system.

- Signal uncertainty: The inability of a CR user to perfectly know the primary signals beforehand that might be present in the band of interest and their properties can be referred as signal uncertainty [87]. The performance of a spectrum awareness technique may depend on the employed standard by the primary system. For example, in an interweave approach, if this information is not known beforehand, the sensing performance may be degraded since a worst case approach is generally followed by the $\mathrm{CR}$ in order to guarantee the target minimum detection performance regardless of the sensed primary signal. Further, signal uncertainty may cause a significant impact on the selection of correct decision threshold in a Constant Detection Rate (CDR) based detector similar to the effect of noise uncertainty in a Constant False Alarm Rate (CFAR) detector [87].

- Noise/Channel correlation: In practical scenarios, the noise may be correlated due to imperfections in filtering, pulse shaping and oversampling in the receiver. Further, the channel may also be correlated due to insufficient scattering in the propagation environment [88] and mutual coupling between antennas [89]. Most of the state of art spectrum awareness methods studied under the assumption of uncorrelated channel and white noise may fail in the presence of noise/channel correlation and there is a need of investigating suitable techniques under practical correlated scenarios [58].

- CR transceiver imperfections: These imperfections are caused by different devices present in the RF chain of a CR. The main transceiver imperfections are discussed below.

1) Amplifier nonlinearlity: Power Amplifier (PA) is a vital component of a CR transceiver and it consumes a large portion of energy in RF circuits during transmission. When operating the PA in the saturation region to achieve the higher efficiency, the nonlin- 
ear distortion increases significantly. This nonlinear behavior of the PA leads to the spectral regrowth of its output signal, resulting in the Adjacent Channel Interference (ACI) [90].

2) Multicarrier distortion: In multicarrier based $\mathrm{CR}$ systems such as in Non-Contiguous Orthogonal Frequency Division Multiplexing (NC-OFDM) systems, high Peak to Average Power Ratio (PAPR) caused by the limited range of the PA and the large spectral sidelobes can be problematic for a $\mathrm{CR}$ system. The high PAPR issue results in serious degradation of Bit Error Rate (BER) performance [91] of the secondary system and the larger side lobes may cause serious performance degradation of the adjacent PUs [92].

3) Quantization errors: Quantization is an important procedure needed to convert the analog RF signal to the digital form with the help of Analog to Digital Converters (ADCs). Quantization may result in the following two kinds of errors: (i) quantization error, and (ii) clipping error. The actual detection performance depends on the quantized samples and may deviate significantly from the performance which is achieved considering ideal samples with infinite precision. Further, clipping error is more problematic for OFDM-like signals having a high PAPR. Moreover, in the low SNR regime, the clipping error may cause a critical problem to detect the weak primary signals. The above quantization errors may cause serious degradation in the detection performance of a CR [93].

4) Synchronization errors (Frequency/time/phase offsets and drifts): For the performance of a secondary system, the secondary receiver needs to be perfectly synchronized with the secondary transmitter in order to carry out demodulation of the transmitted signal. Further, in some detection and estimation approaches which depend on the signal structure of the PU, the ST (CR) has to be perfectly synchronized for the optimum performance. However, in practice, there may occur several types of synchronization errors such as frequeny/time/phase drifts and offsets. In feature based awareness methods, the mismatch in the acquired feature from that of the true feature may cause serious degradation in the awareness performance. For example, cyclostationary feature detector is very sensitive to cyclic frequency mismatch caused by the clock/oscillator or other errors [94]. In the presence of this mismatch, the detector is hardly able to know the exact cyclic frequency where the signal has the cyclostationary feature. Further, I/Q imbalance and nonlinear distortion effects of the receiver components can severely degrade the demodulation performance at weak signal bands, and also affect the reliability of detection reliability of a CR [95].

5) Self interference in full duplex transceivers: A full duplex CR can sense and transmit at the same time, hence maximizing the throughput of the secondary system. However, the main problem in realizing a full duplex transceiver is self interference. It generally employs two RF chains [71] and the signal from its own transmitter may act as an interferer while performing sensing with the help of another RF chain. This self interference needs to be mitigated in practice with the help of suitable RF and digital cancellation methods [96], [97] to a sufficient degree. However, this cancellation may not be sufficiently good in currently available commercial receivers.

In Table II, we list the main imperfections that may affect the performance of three widely used CR paradigms. Although some of these imperfections such as transceiver imperfections, and channel uncertainty also exist in legacy wireless systems, the effect is more serious in CR systems due to the absence of a prior knowledge about the PU system parameters and the channel. Furthermore, since primary and secondary systems may belong to different entities, there exists very low probability of coordination among them in order to acquire these parameters. Among the listed imperfections, noise uncertainty has been widely investigated in the literature and its consequences in CR systems have been known while considering the implementation of a simple energy detector. Further, the existing literature studies the effect of these imperfections individually following different approaches and no common approach/framework is available in the literature to identify which imperfection is more serious over others. In this context, the investigation of a common framework in order to evaluate the effect of these imperfections jointly is an open research problem as highlighted later in Section IX.

In the following sections, we review the contributions carried out in the context of various imperfections from the perspectives of CR systems. Out of these, Sections IV, V, VI are mostly related to the uncertainties that may arise in spectrum awareness phase, Section VII refers to the uncertainty which mostly arise during the spectrum exploitation phase and Section VIII discusses the uncertainties that may occur due to hardware imperfections in a CR transceiver.

\section{NOISE VARIANCE UNCERTAINTY}

In this section, first, we briefly explain why we need to consider SS under noise variance uncertainty. Then, we provide the existing SS algorithms under noise variance uncertainty. As explained previously, the performance of any SS approach is evaluated from its probability of false alarm $\left(P_{f}\right)$ and the probability of detection $\left(P_{d}\right)$. For a given number of samples $(N)$, the $P_{f}$ depends on the statistical characteristics of $z[n]$ (with reference to (1)) and its variance $\sigma^{2}$. On the other hand, the $P_{d}$ expression depends on the statistical characteristics of $z[n], s[n]$ and $h$, and the SNR $\gamma=\frac{\mathrm{E}|s[n]|^{2}}{\sigma^{2}}$. In a practical setup, usually $\sigma^{2}$ is estimated from the received signal (or from the specification of the receiver) and can be bounded as $\sigma_{\min }^{2} \leq \sigma^{2} \leq \sigma_{\max }^{2}$, where $\sigma_{\min }^{2}$ and $\sigma_{\max }^{2}$ are known a priori almost surely. For example, practical receivers have a noise uncertainty level in between 1 to $2 \mathrm{~dB}$ [98], [99]. Under such settings, we can have $\gamma_{\min }=\frac{\mathrm{E}|s[n]|^{2}}{\sigma_{\max }^{2}}$ and $\gamma_{\max }=\frac{\mathrm{E}|s[n]|^{2}}{\sigma_{\min }^{2}}$. 
TABLE II

MAIN IMPERFECTIONS IN DIFFERENT CR PARADIGMS

\begin{tabular}{|c|c|}
\hline $\begin{array}{c}\text { Cognitive Radio } \\
\text { Paradigms }\end{array}$ & Imperfections \\
\hline Interweave & $\begin{array}{ll}\text { - } & \text { Noise uncertainty } \\
\text { - } & \text { Noise correlation } \\
\text { - } & \text { Signal uncertainty } \\
\text { - } & \text { Feature mismatch } \\
\text { - } & \text { CR transceiver imperfections }\end{array}$ \\
\hline Underlay & $\begin{array}{ll}\text { - } & \text { Noise uncertainty } \\
\text { - } & \text { Signal uncertainty } \\
\text { - } & \text { Interference/channel uncertainty } \\
\text { - } & \text { DoA uncertainty } \\
\text { - } & \text { CR transceiver imperfections }\end{array}$ \\
\hline Overlay & $\begin{array}{ll}\text { - } & \text { Channel uncertainty } \\
\text { - } & \text { Feedback errors } \\
\text { - } & \text { CR transceiver imperfections }\end{array}$ \\
\hline
\end{tabular}

In general, the goal of the SS function of a CR network is to ensure sufficient protection level for the PUs. This protection can be maintained if the SS algorithm can detect very low $\mathrm{SNR}$ values (for example, $\mathrm{SNR}=-20 \mathrm{~dB}$ is adopted in Wireless Regional Area Network (WRAN) standard [98], [99]). Conventional Energy Detector (CED) is computationally simple, very easy to implement and does not require any information about the characteristics of the primary transmitted signal. Unfortunately, the authors in [101] revealed that CED is sensitive to noise variance uncertainty and also showed that for a given noise uncertainty level, there exists an SNR wall below which no desired detection performance is guaranteed ${ }^{3}$. As an example, for a $1 \mathrm{~dB}$ uncertainty, the SNR wall of the $\mathrm{CED}$ is around $-3.3 \mathrm{~dB}$ (which is much larger than the required SNR region of WRAN). Due to this fact, a number of research works have been performed to come up with SS algorithms that are robust against noise variance uncertainty which is the focus of the following subsections.

\section{A. Cyclostationary Based Detector}

One of the most distinct behavior of practical communication signals is cyclostationarity [102], [103]. For the received samples $y[n]$, the Cyclic Autocorrelation (CAC) function with cyclic frequency $\alpha$ and time lag $\tau$ is defined as

$$
\hat{R}_{y}^{\alpha}(\tau)=\frac{1}{N_{\tau}} \sum_{n=0}^{N_{\tau}-1} y[n] y[n+\tau]^{\star} e^{-j 2 \pi \alpha n},
$$

where $N_{\tau}=N-\tau$. Under the $H_{0}$ hypothesis, $\hat{R}_{y}^{\alpha}(\tau)=$ $0 \forall \alpha, \tau \neq 0$. However, $\hat{R}_{y}^{\alpha}(\tau) \neq 0$ for some $\alpha, \tau \neq 0$ when there is a transmitted signal $s[n]$, and the exact $\alpha$ and $\tau$ for which the CAC is non-zero depending on different parameters like modulation scheme, symbol period and so on.

In [104] and [105], single cycle and multicycle cyclostationary based detectors are proposed for the hypothesis testing

\footnotetext{
${ }^{3}$ An SNR wall means that a desired $P_{f}$ versus $P_{d}$ target can not be maintained below the SNR wall even if unlimited number of samples are used for sensing.
}

problem (1) by assuming that $s[n]$ is an OFDM signal with the known Cyclic Prefix (CP) factor, and each elements of $z[n], \forall n$ are independent and identically distributed (i.i.d) Zero Mean Circularly Symmetric Complex Gaussian (ZMCSCG) random variables. In particular, the authors in these papers formulate their detection test statistics as the ratio of two quadratic CAC functions [102], [103] where the ratio test is motivated to remove the effect of noise variance uncertainty (i.e., the $P_{f}$ expression will be independent of the noise variance). Also in [106], a ratio of two autocorrelation functions are used to detect the presence of an OFDM signal. For these test statistics, analytical expressions are provided to compute their threshold $\lambda$ for ensuring a prescribed $P_{f}$, and it is shown that $\lambda^{\prime}$ s do not depend on $\sigma^{2}$. And for the given $\lambda$, the $P_{d}$ of these detectors are examined by employing Monte Carlo simulations.

In [107], a sign CAC-based SS approach is used to detect modulated, and OFDM signals with the known CP factor. The main idea of this approach is that each of the received samples is normalized by its modulus. By doing so, the effect of the noise variance is removed effectively under the $H_{0}$ hypothesis. Furthermore, the latter paper shows that such a scaling still maintains the CAC property of modulated (or OFDM) signal whenever there is a primary transmitted signal, and each of the elements of $z[n]$ is i.i.d ZMCSCG random variable. For this approach, the $P_{f}$ is derived analytically whereas, the $P_{d}$ is evaluated numerically using computer simulations.

The cyclostationary based SS approach requires the perfect knowledge of the cyclic frequency and delay lag. And when $\alpha$ is not known perfectly, all possible $\alpha$ s may need to be verified which becomes computationally expensive. However, from the cyclostationary property, for a fixed $\tau$, it is known that most practical signals have non-zero cyclic autocorrelation values for a few values of $\alpha$ s. The authors of [108] exploit this sparsity characteristics to detect the presence of the transmitted signal. In particular, this work proposes to divide the received samples into two groups. And for these groups it determines the most likely cyclic frequency (i.e., corresponding to the largest CAC), denoted by $\alpha_{1}$ and $\alpha_{2}$. Subsequently, it sets $\Delta=1(\Delta=0)$ if $\left|\alpha_{2}-\alpha_{1}\right|<\lambda\left(\left|\alpha_{2}-\alpha_{1}\right| \geq \lambda\right)$, with $\lambda$ being the threshold usually selected based on the desired $P_{f}$, and utilizes the following test statistics

$$
T=\sum_{i=1}^{L_{\tau}} \Delta_{i}
$$

where $\Delta_{i}$ is the $\Delta$ computed when the time lag is set to $\tau_{i}, L_{\tau}$ is the number of lags $\tau_{1}, \tau_{2}, \ldots$, where the CAC is different from 0 .

Additionally, a Receiver Operating Characteristics (ROC) curve has been presented in [108] by employing this statistics. Again in [109], the symmetric properties of the CAC functions are exploited to come up with a blind cyclostationary detector. The key advantage of these contributions is that they utilize a few number of samples and do not need a prior information about the PUs' cyclic frequencies and lags. The latter approach has already been implemented in Universal Software Radio Peripheral (USRP) platform in [110] and has shown promising 
results.

In [111], a blind feature detection approach for wideband spectrum that incorporates energy and cyclostationary is employed. This study uses a combination of the smoothed version of energy detector and the autocoherence function for feature detection. The energy detector is utilized to identify the presence of carrier frequencies in a given sub-band. And the autocoherence function is employed to extract the features of each sub-band such as the number of distinct signals in each carrier frequency. In this regard, first a smoothed version of energy detector is applied to identify whether the carrier frequency contains signal plus noise or noise only signal. Then, when the energy detector declares a given carrier frequency as noise only signal, this information is exploited to determine the appropriate threshold for the autocoherence function of the feature detector to maintain a prescribed false alarm rate for fixed lag $\tau$ and cyclic frequency $\alpha$. To compute this threshold, a simple learning approach that minimizes the Kullback-Leibler distance between the actual and desired false alarm rates has been employed.

\section{B. Autocorrelation based Detector}

Autocorrelation based detectors exploit the difference between the signal and noise spectrums over the sensing bandwidth. This difference arises due to the higher autocorrelation of the signal which may result from the use of practical modulation schemes as well as the presence of RF channel guard bands [112]. In this context, authors in [112] propose a simple Correlation Sum (CorrSum) detector exploiting both energy and correlation parameters for the improved sensing performance assuming that correlation is real and extend to the scenario with the knowledge of correlation distribution information in [113]. Further, a CFAR detection algorithm has been studied in [114] using the estimated autocorrelation of the received signal and its performance is shown to be better than the covariance detector and the cyclic autocorrelation detector.

\section{Covariance based Detector}

This approach is based on the sample covariance matrix of the received signal at the $\mathrm{CR}$ node and exploits the difference in the statistical covariances of the received signal and the noise. It does not require any a priori information of the signal, channel, and noise power and further no synchronization is needed [115]. The authors in [115] propose covariance absolute value and generalized covariance based detection algorithms for a CR. Further, authors in [116] study the distribution of the test-statistic for the covariance based detection and propose analytical expressions for calculating detection and false alarm probabilities.

\section{Eigenvalue based Detector}

This method is based on the Eigenvalue Decomposition (EVD) of the received signal's covariance matrix. For implementing this technique, the $\mathrm{CR}$ node has to collect the received samples in the $M \times N$ matrix form with $M$ being the receive dimension. This receive dimension can be either the number of fractional sampled branches, multiple antennas or the cooperating nodes. A single CR node can implement an eigenvalue-based technique independently if it employs fractional sampling on the received signal and/or is equipped with multiple antennas. After collecting $N$ samples using different receive dimensions, the $M \times N$ received signal matrix $\mathbf{Y}$ can be written as

$$
\mathbf{Y}=\left[\begin{array}{cccc}
y_{1}(1) & y_{1}(2) & \ldots & y_{1}(N) \\
y_{2}(1) & y_{2}(2) & \ldots & y_{2}(N) \\
\vdots & \vdots & \ddots & \vdots \\
y_{M}(1) & y_{M}(2) & \ldots & y_{M}(N)
\end{array}\right]
$$

Let us define the sample covariance matrices of the received signal and the noise as: $\hat{\mathbf{R}}_{\mathbf{Y}}(N)=\frac{1}{N} \mathbf{Y} \mathbf{Y}^{\dagger}$ and $\hat{\mathbf{R}}_{\mathbf{Z}}(N)=$ $\frac{1}{N} \mathbf{Z} \mathbf{Z}^{\dagger}$. Under the $H_{0}$ hypothesis, the sample covariance matrix of the received signal becomes equal to the sample covariance matrix of the noise, i.e., $\hat{\mathbf{R}}_{\mathbf{Y}}(N)=\hat{\mathbf{R}}_{\mathbf{Z}}(N)$.

Different eigenvalue properties of $\hat{\mathbf{R}}_{\mathbf{Y}}(N)$ such as Maximum Eigenvalue (ME) [117], Maximum to Minimum Eigenvalue (MME) or Signal Condition Number (SCN) [36], [98], Energy to Minimum Eigenvalue (EME) [98], Scaled Largest Eigenvalue (SLE) [118], etc. can be utilized in order to perform sensing. Based on these various decision statistics, several eigenvalue based algorithms have been proposed in the literature [98], [119]-[125] exploiting Random Matrix Theory (RMT) methods. These techniques can be broadly categorized into SCN-based [36], [98], [120], [123], ME-based [117], [121], [126] and SLE-based [118], [127], [128]. In addition to the aforementioned methods, spherical test based detection [125], [129]-[131] and John's Detection (JD) [125] have also been considered in the literature. Furthermore, it should be noted that out of a wide range of available eigenvalue based techniques, the techniques such as SCN, SLE, John's detector, spherical test, and EME, which are based on the ratio of the parameters, are robust against noise variance uncertainty.

\section{E. Moment based Detector}

In a digital communication system, the transmitted signal samples are taken from a particular constellation. This constellation may be Binary (Quadrature) Phase-Shift Keying BPSK (QPSK) or M-ary Quadrature Amplitude Modulation (QAM). In all constellations, each component (either real or imaginary) of a sample takes a value in between $[-b, b], b>0$, where $b$ depends on the SNR of the received signal. For these reasons, the contribution in [132] assumes that the transmitted signal samples $s[n], \forall n$ are taken from BPSK, QPSK, M-ary QAM or continuous uniformly distributed random variables ${ }^{4}$ and also assumes that $w[n], \forall n$ are i.i.d ZMCSCG random variables.

Under these assumptions, it is shown in [132] that the ratio of the fourth absolute moment to the square of second absolute moment results 2 and $<2$, under $H_{0}$ and $H_{1}$ hypotheses, respectively [133]. For this reason, a 2 minus the ratio of the fourth absolute moment and the square of second absolute moment is proposed as a test statistics in [132], [133]. For this

\footnotetext{
${ }^{4}$ Note that in the case of OFDM signals, the scenario mentioned in this paragraph can be exhibited by examining the Fourier transform of the received samples.
} 
test statistics, analytical $P_{f}$ and $P_{d}$ expressions are provided. The $P_{f}$ expression does not require the knowledge of $\sigma^{2}$, and the $P_{d}$ expressions differ from one constellation to another.

\section{F. Max-Min SNR based Detector}

1) Max-Min SNR Detector in Time Domain: Some of the characteristics of the primary transmitted signal can be obtained from the regulatory bodies such as FCC. For example, a raised cosine pulse shaping filter has been adopted in Digital Video Broadcasting (DVB) standard [134] with roll off factors $0.2,0.25$ and 0.35 . This motivates [135] to come up with a Max-Min SNR based signal detection approach by exploiting the PT's pulse shaping filter. The main idea of [135] is first to oversample the received signal and introduce a linear combining vector $\boldsymbol{\alpha}$ with size $L$, where $L$ is the oversampling factor. Then, $\alpha$ is optimized such that the combined signal will have two components with different SNRs. Finally, the ratio of the signal energy corresponding to the maximum (which utilizes $\boldsymbol{\alpha}_{\max }$ ) and minimum (which utilizes $\boldsymbol{\alpha}_{\min }$ ) SNRs are used as a test statistics. The $P_{f}$ and $P_{d}$ of this test statistics is derived by applying a simple numerical method which can be computed offline. It is shown that the $P_{f}$ expression depends solely on the receiver's pulse shaping filter, whereas the $P_{d}$ depends both on the transmitter and receivers' pulse shaping filters. On the other hand, for a given $P_{f}$, the best detection performance is achieved when the receiver's pulse shaping filter is the same as that of the PT.

Interpretations of $\boldsymbol{\alpha}_{\min }$ and $\boldsymbol{\alpha}_{\max }$ : The linearly combined oversampled signal by $\boldsymbol{\alpha}_{\max }$ and $\boldsymbol{\alpha}_{\min }$ will achieve different SNRs under the $H_{1}$ hypothesis. The interesting question is why? When we introduce the linear combination term $\boldsymbol{\alpha}$, we naturally modify the overall filter at the receiver. By going into the details of the Max-Min SNR algorithm, one can notice that the modified filter will have the following coefficients

$$
\begin{aligned}
\mathbf{A} & =\operatorname{diag}(\boldsymbol{\alpha}) \mathbf{f} \\
c_{i} & =\sum \operatorname{diag}(\mathbf{A}, i) \\
\mathbf{c} & =\left[c_{-L+1}, c_{-L+2}, \cdots, c_{L_{f}-1}\right],
\end{aligned}
$$

where $\mathbf{f} \in \mathcal{C}^{L_{f} \times 1}$ is the nonzero values of $f(\tau)$ sampled at a rate $\frac{L}{P_{s}}$, with $P_{s}$ being the symbol period of the transmitted symbols, and $\sum \operatorname{diag}(\mathbf{A}, i)$ represents the sum of the $i$ th diagonal elements of $\mathbf{A}$, with $i=0$ denotes the main diagonal elements and $i<0(i>0)$ denotes the left (right) diagonal elements.

When we employ $\boldsymbol{\alpha}_{\min }$ and $\boldsymbol{\alpha}_{\max }$, we will have the corresponding $\mathbf{c}_{\min }$ and $\mathbf{c}_{\max }$, respectively. Figure 7 shows the spectrum of $\mathbf{c}_{\min }$ and $\mathbf{c}_{\max }$ when $f(\tau)$ is a Square Root Raised Cosine Filter (SRRCF) with bandwidth $B$ and excess bandwidth $\beta$. As we can see from this figure, the spectrum of $\mathbf{c}_{\max }$ amplifies the received signal in the bandwidth $\left[-\frac{B}{2}, \frac{B}{2}\right]$ and attenuates the rest band whereas, $\mathbf{c}_{\min }$ amplifies the received signal in the region $\pm\left[\frac{B}{2}, \frac{B(1+\beta)}{2}\right]$ and attenuates the rest. This observation is reasonable as the maximum SNR is achieved in $\left[-\frac{B}{2}, \frac{B}{2}\right]$ (i.e., the energy of the pulse shaped signal is concentrated in this frequency range).

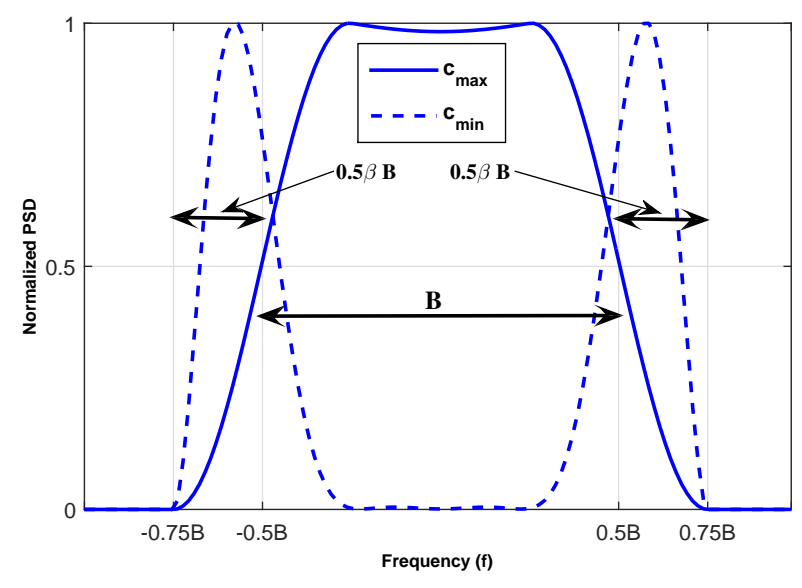

Fig. 7. The spectrum of $\mathbf{c}_{\max }$ and $\mathbf{c}_{\min }$.

2) Max-Min SNR Detector in Frequency Domain: The work of [135] (i.e., Max-Min SNR Detector in Time Domain) is performed by employing the time domain samples and it requires oversampling of the received signal with $L \geq 8$ which is not desirable in practice as such operation requires a higher rate $\mathrm{ADC}$ at the cognitive receiver. Furthermore, the spectrum of $\mathbf{c}_{\min }$ and $\mathbf{c}_{\max }$ do not necessarily have constant spectral gain in their regions of interest (i.e., in $\pm\left[\frac{B}{2} \frac{B(1+\beta)}{2}\right]$ for $\mathbf{c}_{\min }$, and $\left[-\frac{B}{2}, \frac{B}{2}\right]$ for $\left.\mathbf{c}_{\max }\right)$. However, from the work of [135], one can notice that the frequency band $\pm\left[\frac{B}{2} \frac{B(1+\beta)}{2}\right]$ contains almost noise information. And the band $\left[-\frac{B}{2}, \frac{B}{2}\right]$ contains the transmitted signal under $H_{1}$ hypothesis. For this reason, one can modify $\mathbf{c}_{\max }$ and $\mathbf{c}_{\min }$ such that their spectral gains are constant in the region of interest. This motivates [136] to employ the test statistics utilizing the ratio of the energy of the frequency band $\left[-\frac{B}{2}, \frac{B}{2}\right]$ and that of $\pm\left[\frac{B}{2} \frac{B(1+\beta)}{2}\right]$. In fact, the detection approach of [136] can be considered as the frequency domain version of [135]. Hence, despite the test statistics of [135], the test statistics used in [136] requires the excess bandwidth $\beta$ only (i.e., the test statistics used in [136] does not require oversampling of the received signal and knowledge of the pulse shaping filter coefficients).

\section{G. Generalized Energy Detector (GED)}

To get more insight about the work of [136], we consider a more general scenario where we have two sub-bands $B_{1}$ and $B_{2}$, where $B_{1}$ (for example the frequency region $\pm\left[\frac{B}{2} \frac{B(1+\beta)}{2}\right]$ in Fig. 7) contains noise information only, and $B_{2}$ (for example $\left[-\frac{B}{2}, \frac{B}{2}\right]$ in Fig. 7) may contain noise only and signal plus noise information under the $H_{0}$ and the $H_{1}$ hypotheses, respectively. The two sub-bands $B_{1}$ and $B_{2}$ can also be illustrated as shown in Fig. 8.

Let $N_{T}$ be the number of samples obtained by employing the Nyquist rate on the received signal of Fig. 8 (i.e., sampling rate of $B_{T}=B_{1}+B_{2}$ ). For this setting, the following test statistics is proposed in [136] to detect sub-band 1

$$
T=\sqrt{\frac{N_{1} \tilde{\beta}}{\tilde{\beta}+1}}\left(\frac{\mathrm{AE}\left(\left[0: B_{1}\right]\right)}{\operatorname{AE}\left(\left[B_{1}: B_{T}\right]\right)}-1\right),
$$




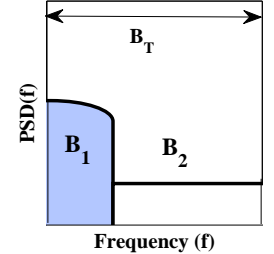

Fig. 8. Two Sub-bands: One desired and one white sub-bands.

where $N_{1}=\frac{B_{1}}{B_{T}} N_{T}, \tilde{\beta}=\frac{B_{2}}{B_{1}}$ and $\operatorname{AE}([x: y])$ denotes the average energy in the frequency region $[x: y]$. For the better exposition of the test statistics (6), let us examine $T$ for the fixed $B_{1}$ and $\tilde{\beta} \rightarrow \infty$ as

$$
T_{\tilde{\beta} \rightarrow \infty}=\sqrt{N_{1}}\left(\frac{\mathrm{AE}\left(\left[0: B_{1}\right]\right)}{\sigma^{2}}-1\right),
$$

where $\operatorname{AE}\left(\left[B_{1}: B_{T}\right]\right)=\sigma^{2}$ when $B_{2} \rightarrow \infty$. Indeed, this is a shifted and scaled version of the CED. From this, we can understand that the term $\mathrm{AE}\left(\left[B_{1}: B_{T}\right]\right)$ in (6) is introduced just to estimate the noise variance. Hence, the test statistics (6) can be regarded as a Generalized Energy Detector (GED) [99]. From (7), one can understand that perfect noise variance has the same significance as having "infinite" bandwidth to estimate the noise variance.

From the above explanation we can see that if $B_{2} \rightarrow \infty$, the performance of the GED is the same as that of the CED. So the interesting question will be how much the performance loss of the GED is compared to that of the CED when $B_{2}$ is finite (i.e., $\tilde{\beta}$ is finite) which is the case in practice. The detection performance loss is provided in [99] as

$$
\eta=1-\frac{\operatorname{erfc}\left(\frac{\lambda^{\star}-\sqrt{\frac{N_{1} \tilde{\beta}}{\tilde{\beta}+1}} \gamma}{\sqrt{2}(1+\gamma)}\right)}{\operatorname{erfc}\left(\frac{\lambda^{\star}-\sqrt{N_{1}} \gamma}{\sqrt{2}(1+\gamma)}\right)},
$$

where erfc(.) is the complementary error function. From this expression, one can notice that $\eta \rightarrow 0$ (i.e., almost no loss) when $\tilde{\beta} \approx 10$. Therefore, if there is a sufficient bandwidth to estimate the noise variance (i.e., $\approx 10 B_{1}$ ), perfect noise variance information is not a limiting factor for energy detector. Furthermore, this sufficient bandwidth is relative to the desired sub-band (i.e., $B_{1}$ ).

The GED can be implemented when there is a sub-band that contains noise only like in the considered example in this subsection. However, how can we implement the GED when the sub-band that contains noise only information is not known a priori? For such a scenario, the work of [99] first applies an edge detector to identify the edges of all sub-bands. Then, a reference white sub-band detection is used to reliably detect the sub-band that contains noise only information (it is termed as a reference sub-band [99]). Finally, the GED of (6) is employed to detect each of the sub-bands other than the reference sub-band. The detailed implementation of [99] can be found in [100].

Figure 9 shows the comparison of cyclostationary, EVD, Moment and Max-Min SNR based SS algorithms with noise variance uncertainty. For EVD, Moment and Max-Min SNR

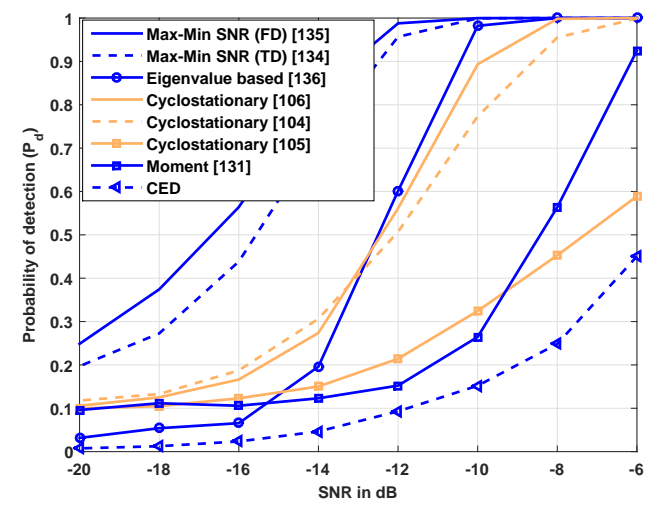

(a)

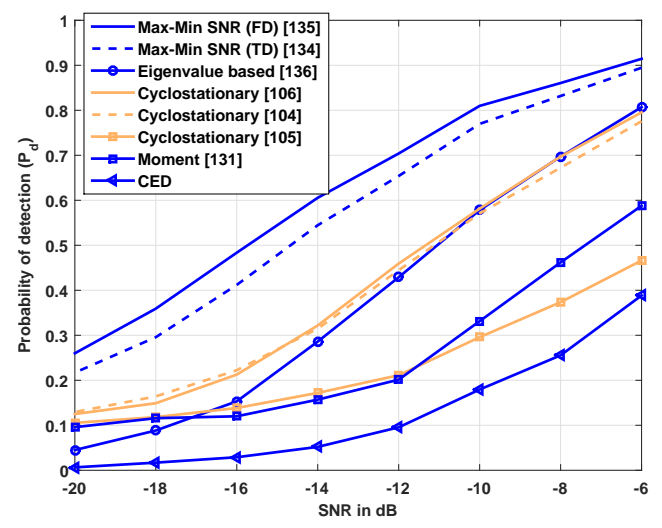

(b)

Fig. 9. Comparison of different algorithms under noise variance uncertainty $\left(P_{f}=0.1, N=2^{15}, \triangle \sigma^{2}=2 \mathrm{~dB}\right.$, QPSK). The FD (TD) stands for frequency (time) domain. Here, Max-Min SNR (FD) [136], Max-Min SNR (TD) [135], Eigenvalue based [137], Cyclostationary [107], Cyclostationary [105], Cyclostationary [106], Moment based [132], (a) AWGN channel, (b) Rayleigh fading channel.

based SS algorithms, we assume a QPSK transmitted signal which is pulse shaped by a root raised cosine filter with roll off factor of 0.2. The number of received samples is set to $N_{1}=2^{15}=32768$ (i.e., with Nyquist sampling). And for the cyclostationary based detectors, we consider an OFDM signal with the settings as in Table I of [105] but $N_{\text {OFDM }}=57$. By doing so, the cyclostationary detectors will utilize $N_{2}=$ $32832 \approx N_{1}$ samples when Nyquist sampling is applied. As we can see from this figure, the frequency domain version of the Max-Min SNR based SS algorithm (i.e., GED for pulse shaped signals) achieves the best performance among all, and as expected the worst performance is obtained with the CED.

In Fig. 9, we have compared different SS techniques for a common setting in which all the considered detectors employ $P_{f}=0.1, \Delta \sigma^{2}=2 \mathrm{~dB}$ and almost the same sensing durations. However, different techniques generally take different assumptions/parameters into account and their corresponding performance depends on the employed assumptions/parameters. Due to this reason, comparing different SS techniques just by 
enforcing the same $P_{f}, \Delta \sigma^{2}$ and the sensing time may not be a general comparison strategy. Consequently, the Max-Min SNR (FD) approach achieves the best performance only for the setting of this paper, and the extensive comparison of different $\mathrm{SS}$ approaches for various scenarios is still an open research topic.

\section{NoIse/ChAnNel CORRELATION}

Most of the existing SS and SNR estimation techniques assume the presence of an uncorrelated channel and the noise [56], [98], [138]. However, in practical scenarios, the channel may be correlated due to insufficient scattering in the propagation environment [88] and mutual coupling between antennas [89]. Similarly, the noise may also be correlated due to imperfections in filtering, pulse shaping and oversampling in the receiver. The main causes behind noise and channel correlation are listed in Table III. The correlation may arise across the receive dimensions, i.e., spatial correlation, and/or across the samples acquired by a single receive dimension, i.e., temporal correlation. Further, as stated earlier, a CR is not aware of the PU signal, channel and the noise variance. Therefore, one important research issue is to investigate blind SS and SNR estimation techniques which can effectively work in practical correlated scenarios. In this context, authors in [36] have recently analyzed the effect of noise correlation on eigenvalue based SS and have shown that the existing decision thresholds fail in the presence of noise correlation.

To address the aforementioned issue, new sensing thresholds for SCN and ME based techniques have been proposed in [139], [140] using recent results from RMT in order to achieve the improved sensing in correlated noise scenarios. The analysis has been carried out by considering a one-sided exponential correlation model to include noise correlation across the receive dimensions and the similar analysis is applicable for temporally correlated case assuming the exponential correlation model still holds. As reflected in Fig. 10, the sensing with the conventional Marchenko Pasteur (MP) based decision threshold [119] decreases drastically with the increased amount of noise correlation while the proposed sensing bounds in [139] provide better performance up to some value of noise correlation. Moreover, it has been noted that new sensing bounds also do not provide better sensing performance at the high correlation region due to the larger overlapping of the the distributions of test statistics under the $H_{1}$ and $H_{0}$ hypotheses at this region.

As noted in Section IV-D, several blind eigenvalue based techniques such as SCN-based detector, SLE-based detector, John's Detector (JD), and spherical test detector exist in the literature. Most of these detectors fail to provide better sensing performance in the presence of noise correlation [35]. Figure 11 shows the effect of noise correlation on different blind eigenvalue based techniques and it can be noted that JD detector has the worst performance among all the techniques and the SLE detector is the best in terms of sensing performance in the presence of correlated noise.

Moreover, most of the multi-dimensional SS techniques proposed in the literature do not consider the effect of channel

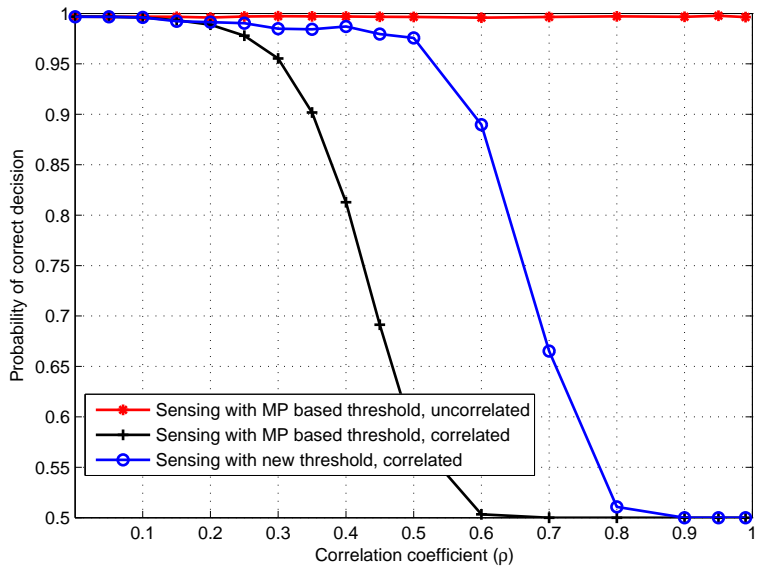

Fig. 10. Sensing performance versus correlation coefficient $\rho$ $(\mathrm{SNR}=-6 \mathrm{~dB}$, Number of samples $(N)=60$, Number of receive dimensions $(M)=10)$ [36]. The performance metric "Probability of correct decision" depicts how many correct decisions are made out of the total considered realizations under both hypotheses.

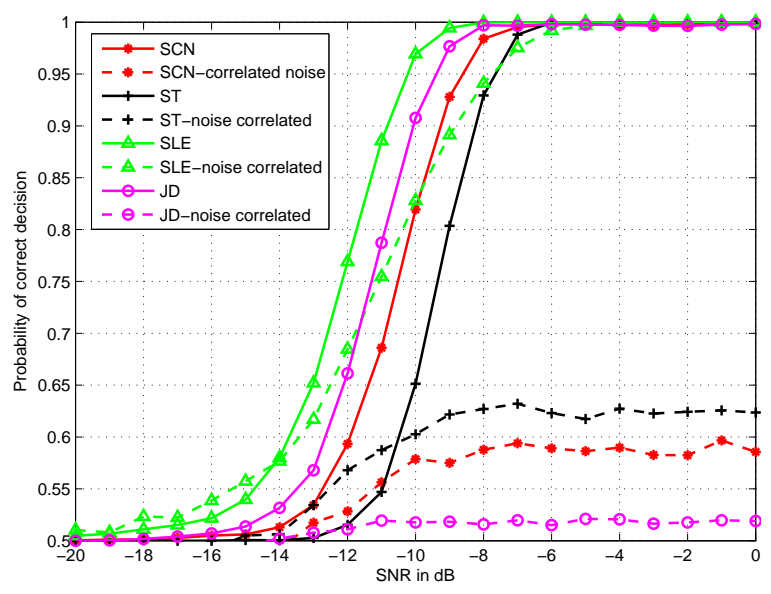

Fig. 11. Sensing performance versus SNR for different eigenvalue based blind techniques in the presence of correlated noise ( $M=$ $10, N=100, \rho=0.45)$ [35]. The SCN stands for Signal Condition Number, ST for Spherical Test, SLE for Scaled Largest Eigenvalue, and JD for John's Detector.

correlation. Some contributions in the literature have analyzed the performance of the ED technique with multiple antennas considering spatial correlation across these antennas [141][143]. The authors in [141] analyzed the sensing performance of an energy detector with correlated multiple antennas and it was verified that the sensing performance of the energy detector is degraded when the channels are spatially correlated and the performance degradation is proportional to the level of correlation. Furthermore, the authors in [142] analyzed the detection performance of an ED based SS in a CR with multi-antenna correlated channels in the Nakagami$\mathrm{m}$ fading channel. Additionally, a weighted ED technique and a correlated Generalized Likelihood Ratio Test (GLRT) detector have been proposed in [143] for SS with multiantenna correlated channels. In the context of eigenvalue-based $\mathrm{SS}$, the effect of spatial correlation in the performance of predicted eigenvalue threshold based SS is analyzed in [122] and it is shown that the detection performance improves in 
TABLE III

CAUSES OF CHANNEL/NOISE CORRELATION IN MULTI-DIMENSIONAL SCENARIOS [58]

\begin{tabular}{|c|c|c|c|c|}
\hline \multirow[t]{2}{*}{ Cases } & \multicolumn{2}{|c|}{ Channel Correlation } & \multicolumn{2}{|c|}{ Noise Correlation } \\
\hline & Type & Cause & Type & Cause \\
\hline \multirow{2}{*}{$\begin{array}{l}\text { Multi-antenna } \\
\text { SS }\end{array}$} & Spatial Correlation & $\begin{array}{l}\text { Multipath } \\
\text { propagation }\end{array}$ & \multirow[t]{2}{*}{ Filtering } & \multirow{2}{*}{$\begin{array}{l}\text { Autocorrelation } \\
\text { function of the } \\
\text { filter }\end{array}$} \\
\hline & $\begin{array}{l}\text { Antenna mutual } \\
\text { coupling }\end{array}$ & $\begin{array}{l}\text { Transmit/receive } \\
\text { Antenna } \\
\text { separation }\end{array}$ & & \\
\hline \multirow[t]{2}{*}{ Oversampled SS } & Oversampling & $\begin{array}{l}\text { Oversampling } \\
\text { operation }\end{array}$ & Filtering & $\begin{array}{l}\text { Autocorrelation } \\
\text { function of the } \\
\text { filter }\end{array}$ \\
\hline & Filtering & $\begin{array}{l}\text { Autocorrelation } \\
\text { function of filter }\end{array}$ & Oversampling & $\begin{array}{l}\text { Oversampling } \\
\text { operation }\end{array}$ \\
\hline Cooperative SS & Spatial correlation & $\begin{array}{l}\text { Collocated nodes in } \\
\text { CR network }\end{array}$ & Filtering & $\begin{array}{l}\text { Autocorrelation } \\
\text { function of the } \\
\text { filter }\end{array}$ \\
\hline
\end{tabular}

the presence of spatial correlation at the multi-antenna SU. Similar trend has been recently obtained in [58] for the case of SCN and EME based detectors.

As noted earlier in Section II-A, various data-aided and non-data-aided SNR estimators have been investigated for traditional legacy based systems. SNR estimation for legacy based systems is helpful for implementing adaptive techniques such as adaptive bit loading, handoff algorithms and optimal soft value calculation for improving the performance of channel decoders. In addition to these benefits, estimation of primary SNR is important for CR-based systems in order to design their underlay transmission strategies [58]. In practical CR scenarios, there is a need of blind SNR estimation techniques which can effectively work under practical correlated scenarios.

In the above context, a novel eigenvalue based SNR estimation technique has been recently studied in [58] under noise/channel correlated scenarios using the RMT tool. From the presented results in [58], it can be noted that the effect of channel correlation dominates at higher SNR values while the effect of noise correlation dominates at low SNR values. Additionally, the SNR estimation problem for a wideband cognitive receiver has been studied in [144] under correlated noise and correlated multiple measurement vector scenarios. A tradeoff between sensing hardware cost (especially the cost of the $A D C$ ) and the estimation error has been observed while using compressive measurements. Furthermore, it has been concluded that correlation knowledge significantly helps while estimating the SNR under correlated scenarios.

\section{SignAl UNCERTAINTY}

It can be noted that most of the SS contributions in the literature assume the known signal distributions while deriving the analytical expressions for $P_{d}$ and $P_{f}$. However, in practice, due to time varying nature of wireless channels, it is usually very challenging to know precise information regarding PU signals' probability distribution. For example, the received signals may exhibit different distributions depending on whether there is a Line of Sight (LoS) channel between primary and secondary systems or a scattering channel. Further, the mobility of wireless nodes also affects the signal distributions significantly. In this context, authors in [145] study lower and upper bounds of sensing performance of an energy detector based on different signal uncertainty models. Various uncertainty models considered in [145] are: (i) moment uncertainties that are based on moment statistics, e.g., sample mean and variance estimates of the received signals, (ii) distribution information embedded in the historical data, from which a reference distribution can be extracted using the goodness of fit test, and (iii) uncertainty model in which the actual signal distribution is allowed to fluctuate around a reference distribution and the discrepancy is characterized in terms of a probabilistic distance measure.

Although noise uncertainty aspect has been analyzed and studied in various literature, the aspect of signal uncertainty has received limited attention. The recent contribution in [87] analyzes the impact of signal uncertainty on the detection performance of the ED approach considering a signal uncertainty model. From the results, authors have shown that the signal uncertainty phenomenon may result in significant performance degradations, particularly in the presence of noise uncertainty even for very small noise power calibration errors. Furthermore, authors in [87] define signal uncertainty as the inability of a CR user to perfectly know the information about primary signals beforehand. This uncertainty may lead to the degradation in the sensing performance since the noninterference requirement for the secondary network implies a worst-case design where SUs must guarantee a minimum detection performance regardless of the sensed primary signal.

\section{Channel/Interference UnCERTAinty}

Channel/interference uncertainty is of significant importance in the context of an underlay CR. Figure 12 presents 


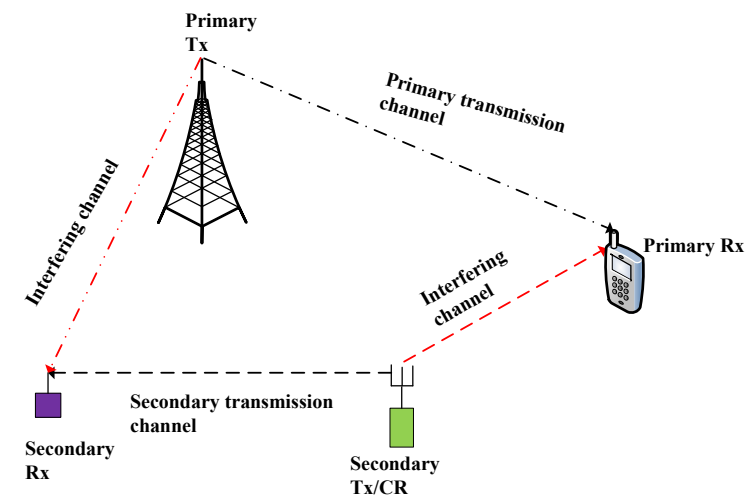

Fig. 12. A typical underlay coexistence scenario with data transmission and interfering links

a typical underlay coexistence scenario with transmission and interfering links. Under this scenario, the main challenge for a $\mathrm{CR}$ is to control its transmission power in order to optimize its Quality of Service (QoS) while limiting the interference to the PUs below the tolerable interference limit. While studying the considered coexistence scenario, the channel uncertainty may arise either in the interfering link between the ST/CR and the PR or in its desired transmission link towards the $\mathrm{SR}^{5}$. From the regulatory point of view, the interfering link between the ST/CR and the PR is of more importance than the interfering link between the PT and the CR since a CR system can be assumed to be intelligent enough to mitigate the interference from the primary system. On the other hand, from the secondary system's perspective, the channel between the ST and the SR is of importance in order to guarantee the desired QoS of the link. In practice, this underlay coexistence can be realized using several approaches such as power control, EZ, and dynamic resource allocation.

One widely used approach of controlling the SUs' transmission is to control the transmit power at the CR by estimating the channel $h$, in (1), towards the PU based on the estimation of the received primary signal $y$. In most of the cases, SS is performed by carrying out analysis under the $H_{0}$ hypothesis as discussed in Section IV whereas the received signal power estimation is carried out under the $H_{1}$ hypothesis. In this received power estimation based power control approach, the main problem is that the PR may be a passive entity and the channel reciprocity may not be applicable in Frequency Division Duplex (FDD) based systems. However, there exist several practical scenarios in which secondary transmission can be controlled based on the estimated primary power at the CR [24]. These scenarios are briefly described below.

1) Beacon-based transmission: This scenario considers the beacon-based sensing, in which a PR is assumed to send a beacon signal on a control channel [146]. In this context, the SUs can detect the presence of a $\mathrm{PR}$ as well as estimate the power of the beacon signal. Subsequently, based on the strength of the beacon signal

\footnotetext{
${ }^{5}$ Herein, the assumption is that the uncertainty in the transmission channel of the primary system is already taken into account while designing the primary system.
}

and interference threshold of the PU, the SU can adjust its transmit power in order to protect nearby primary receivers.

2) Duplex transmission: This case assumes duplex transmission mode for the PUs, i.e., each user interchangeably transmits and receives over time under the Time Division Duplex (TDD) mode. In this scenario, the CR estimates the received PU power when it is operating in the receive mode and controls its transmitted power during its transmission based on the estimated received power utilizing channel reciprocity principle [71], [148]. If there exist multiple PUs in the system, we need to consider the worst case condition. For a number of PUs under a given power class, if a PU with the minimum path loss, i.e., strongest estimated power is protected, all other PUs under the considered class can be protected at the same time assuming the same interference threshold. The main problem with the above worst case approach is that it may not be possible to estimate the received SNR of all the PUs. In this context, another solution to address the problem of multiple PUs protection can be the design of a power control algorithm based on the aggregate received power instead of the strongest received power.

3) Short-range simplex transmission: This scenario considers the simplex mode of transmission for the PUs and a short range wireless communication for both primary and secondary systems provided that interference levels from one system to another are at a similar level. In practice, this case arises when a spectrum resource is left completely unused within a sufficiently large network coverage area [147]. Another scenario could be the operation of primary and secondary systems in indoor and outdoor environments [148]. In this case, fixing secondary transmit power based on the received signal from the PT is a reasonable strategy for protecting the PR as well.

4) Detection margin based secondary coverage: In this scenario, the protection of the PRs can be provided by including a detection margin in defining the coverage region of a CR [146]. For example, let $r_{p}$ and $r_{s}$ denote the maximum communication range of the primary system and the interference range of the CR. In this case, the CR should be capable of detecting any active PT within a radius of $r_{p}+r_{s}$ in order to guarantee that no active PRs exist within its interference range.

We present an example for the uncertainty in the interfering channel between the $\mathrm{CR}$ and the PR considering the power control based approach below. Let $I_{T}$ be the interference threshold of the PR, which can be obtained from the regulatory requirements, i.e., based on the interference protection criteria specified in the regulations for a particular primary system. In an ideal case, the transmit power for a ST $P_{\mathrm{st}}$ can be determined based on the following interference protection criteria

$$
P_{\mathrm{st}} \leq \frac{I_{\mathrm{T}}}{\alpha_{p} h_{p}}
$$

where $I_{T}$ denotes the interference threshold for the PR, $\alpha_{p}$ 
corresponds to the distance dependent path loss and $h_{p}$ denotes the fading coefficient due to the time-varying channel. In practice, the value of $\alpha_{p} h_{p}$ in (9) can be calculated with the help of the estimated PU SNR and the Effective Isotropic Radiated Power (EIRP) of the primary system which can be obtained based on the specifications of wireless standards such as LTE, GSM/GPRS, EDGE etc [71]. From (9), it can be deduced that the value of $\alpha_{p}$ is fixed but the variation in the value of $h_{p}$ may lead to the violation of the interference criteria at the PR. Therefore, it is significantly important to capture the effect of channel uncertainty while estimating the received primary power at the $C R$.

In most of the underlay CR techniques, the SUs need Channel State Information (CSI) towards PRs in order to avoid harmful interference to the PRs. This information can be static or dynamic depending on the propagation environment. However, in practice, there is no coordination between primary and secondary systems in exchanging the CSI. Due to this reason, the secondary system may not be able to obtain the CSI information towards the primary system accurately and the channel estimation techniques have to rely on power based estimation. Further, atmospheric imperfections such as smallscale fading, shadowing may create great uncertainty in the CSI. Therefore, interference awareness based approaches may not be reliable in practice. The channel estimation in CR systems is challenging due to the following aspects

- CRs can access to the shared band only intermittently

- PU system often does not explicitly support channel estimation for CR systems

- CRs might not have prior knowledge of PU signal characteristics

- PU transmissions must be strictly protected from the interference due to CRs, thus leading to the need of accurate channel estimation.

To perform the accurate channel estimation, physical channel conditions should remain stable for a sufficient period. However, in practice, such a stable condition may be very short due to user mobility and the SUs may not be able to detect the channel reliably [149]. Therefore, channel estimation can be never perfect in practice and estimation errors need to be taken into account while designing underlay strategies for a CR. Besides, channel uncertainty may also originate from the lack of regular information exchange between primary and secondary systems. As a result, the interference information at the PRs can not be fed back to the STs on time. Further, due to the time-varying nature of the wireless fading channel as well as possible hidden nodes in the wireless network, it is generally challenging for a single CR to accurately obtain the instantaneous system parameters in order to devise the best transmission strategy. In the above context, several contributions in the literature have studied the performance of cognitive resource allocation problems taking account of channel estimation errors.

Based on the perfect and imperfect channel knowledge, the available approaches in the literature can be broadly categorized into (i) robust and (ii) non-robust methods. The robust (non-robust) methods take (do not take) into account of the channel estimation uncertainty while designing power control/resource allocation strategies at the receiver. The existing approaches for analyzing the effect of channel uncertainty are described below.

1) Theoretical Model based Estimation: Some existing works assume a prior knowledge of PUs' locations or spatial distribution, based on which SUs can estimate the interference at the PRs using a predefined propagation model. In this context, the contribution in [150] characterizes the impacts of SUs' transmission power on the occurrence of spectrum opportunities and the reliability of opportunity detection. Based on a Poisson model of the primary network, authors in [150] have studied these impacts by showing the exponential decay rate of the probability of opportunity with respect to the transmission power and asymptotic behavior of the ROC curve for the spectrum opportunity detection. However, the methods based on the predefined propagation model may not be practical as they usually require a site survey before the deployment of a secondary network. Besides, the interference estimation based on theoretical propagation models is generally oversimplified and may significantly differ from reality.

2) Channel Reciprocity: Another method for dealing with the channel uncertainty is to estimate the reciprocal channel by overhearing feedback from the PRs [151]. Authors in [151] propose a framework that incorporates the inherent feedback information in typical two-way PU communication links such as power control feedback in Code Division Multiple Access (CDMA) cellular, channel quality indicator feedback in HSDPA systems, ACK/NAK feedback in cellular or WiFi networks etc. Such feedback information from the PR can provide a good indicator of the actual impact of the SUs' interference on the reception quality of the PU communication link. Due to the limited observations of fedback from the PRs, this method may be unreliable for real-time power control. More specifically, PRs may send feedback in the form of ACK packets sporadically after receiving a bulk of data streaming and channel estimation as this approach may not capture the variations of channel characteristics accurately. If power control is based on outdated channel estimates, SUs cannot know the actual interference at the PU receivers, thus leading to violations of PUs' interference constraints in time varying channel conditions.

3) Channel Uncertainty Modeling: The third approach is based on excessive interference avoidance caused by channel uncertainty. One of the widely used methods is to model the channel gain as a combination of deterministic and random components. The available related literature in this paradigm can be categorized into the following three categories

- Stochastic Approach: This approach assumes the random component to follow a certain distribution function, which usually leads to chance constraints in the power control problem [152], [153]. The main 
drawback of this approach lies on the assumption of a known distribution function, which is either unavailable or is very complex to obtain in practice.

- Worst Case Approach: This approach allows to restrict the randomness of the random component within a bounded and convex set [154], [155]. This approach can provide the highest PU protection level but may result in conservative performance for the secondary link. Further, another difficulty lies in finding an appropriate bounding set to model the uncertainty of the random component.

- Reference Distribution based Approach: To address the above drawbacks of the worst-case and stochastic based approaches, authors in [149] recently proposed a reference distribution based channel uncertainty model in which the reference distribution is extracted from historical channel measurements. The actual distributions of aggregate interference power and SINR are allowed to deviate from their reference distributions by a probabilistic distance measure considering various uncertain factors. Subsequently, a power control problem has been formulated as a chance constrained robust optimization which takes distribution functions as the uncertain variables, and an iterative algorithm has been developed in order to search for the optimal transmit power.

The investigation of suitable methods in order to provide robustness against channel/interference uncertainty has recently received a lot of attention, especially in the following two contexts: (i) robust power control for Single Input Single Output (SISO) CR networks [149], [153], [156], [157], and (ii) robust beamforming for MIMO or Multiple Input Single Output (MISO) CR networks [152], [154], [155], [162]. We describe important design issues for these scenarios below.

1) Robust Power Control for SISO CR Networks: An important aspect to be considered in the implementation of an underlay CR network is how to dynamically allocate the secondary transmit power in order to maximize the secondary throughput while providing sufficient protection to the PRs. This problem has been widely studied in the literature in various settings [149], [153], [156], [157]. This can be basically formulated as the maximization of the SUs' social utility subject to three constraints, namely, a given PU interference threshold, the SUs' minimum requested SINR, and the SUs' upper bound on their transmit power levels. In this problem, the main uncertain parameters to be considered are : (i) uncertainty in the channel gains between SUs and PRs, and (ii) uncertainty in the interference from PUs to the SRs. Besides, several practical issues need to be considered while designing a power control mechanism for a CR as described below.

In a CR network, new users may join the network, or existing users may leave the network at any instant of time. Furthermore, the PUs may start or stop communication, and hence they may occupy or release some spectrum bands in an uncertain manner. All these occurrences can be considered as discrete events compared to the realtime evolution of each user's power vector, which can be generally considered as evolving in continuous time. Therefore, the CR design problem can be considered as a mixture of continuous dynamics and discrete events [157]. In this context, several issues such as statistical behavior of the PUs, channel transition probabilities, model uncertainty etc. need to be considered while designing robust power control methods for CR networks. In practice, the statistical behavior of the PU as well as the channel transition probabilities are difficult to characterize accurately. Moreover, due to time-varying nature of the wireless channel and the required sensing overhead, only the delayed SS results may be available for the decision process. In addition, due to the mobility and the limited battery capacity of the CRs, power control should take into account the time-varying nature of the wireless channel in order to efficiently utilize the available transmission power [156].

In fast fading wireless environments, a CR should be able to perform channel sensing in much shorter time intervals to catch up with the fast variations. In this case, the $\mathrm{CR}$ is not able to make a reliable decision about the PU activity only from a single test statistic over the sensing result because of the short sensing duration. To improve the decision process in such environments, Partially Observable Markov Decision Process (POMDP) framework [158], which helps to automate the decision making process by using the channel state dependent policy or function, has been investigated in several literature [159]-[161]. The statistical-based sensing model used in this framework employs a probabilistic approach rather than the deterministic approach while designing optimal decision-making algorithms [160].

2) Robust Cognitive Beamforming for MIMO/MISO CR Networks: The main difference between a conventional beamforming problem and the cognitive beamforming problem is the introduction of interference constraints imposed by the primary network while designing the beamformer. These constraints may greatly increase the complexity of the corresponding beamforming and resource allocation techniques. Recently, cognitive beamforming has been widely studied with different secondary network optimization objectives such as sum rate maximization, SINR/rate balancing, and power minimization with QoS constraints (See [154] and references therein). Similar to conventional beamformers, a cognitive beamformer should be robust against the desired user's DoA mismatch, array steering vector and channel uncertainties.

The non-robust cognitive beamforming approaches proposed in the literature are based on the assumption of the perfect CSI/DoAs in the directions of PRs and SRs at the SU transmitter, which are usually difficult to achieve due to limited training, less cooperation between $\mathrm{SU}$ and PU, and quantization issues. Furthermore, in practice, the estimates of steering vectors are prone to 
errors either due to incorrect estimates of the DoA, or due to antenna-array imperfections. In this context, the worst-case [154] and stochastic [152] methods have been proposed to tackle the robust cognitive beamforming problem. However, these studies only consider stationary SUs and a general robust design study applicable to both stationary and mobile SRs in practical scenarios is limited [163]. In this context, the recent contribution in [163] has proposed a transmit beamforming scheme that is robust against errors in steering vector estimations for the underlay $\mathrm{CR}$ system with mobile or stationary SR and multiple PUs in different locations using an stochastic optimization method.

Besides the effect of channel uncertainty in underlay based CR systems, channel uncertainty in the sensing channel of a $\mathrm{CR}$ operating under the interweave mode may also provide a significant effect. As mentioned earlier in Section III, channel uncertainty may affect in the choice of a correct decision threshold in a CDR-based detector. Furthermore, in the cooperative SS scenario presented in Fig. 5, practical limitations of the fusion rule at the Fusion Center (FC) need to be considered carefully. One of the main issues in this scenario is imperfect reporting channels between the secondary nodes and the FC. Most of the literature assumes the error-free reporting links but this is not the case in practice. Similar to the concept of SNR wall in case of an energy detector, there exists the concept of Bit Error Probability (BEP) wall which limits the performance of cooperative sensing system [164], [165]. If the $B E P$ of the reporting channel is higher the BEP wall value, it is impossible to satisfy the imposed performance constraints on the detection error probabilities at the FC irrespective of the sensing time at the SUs or the SNR on the sensing channel.

\section{CR TRANSCEIVER IMPERFECTIONS}

\section{A. Power Amplifier Nonlinearity}

As noted earlier, interference characterization and mitigation are critical for a CR while dealing with resource allocation problems. One of the important issues to be considered is the nonlinearity of the Power Amplifier (PA). The PA consumes a large portion of energy in RF circuits during transmission and when operated in the saturation region to achieve the higher efficiency, the nonlinear distortion increases significantly. This nonlinear behavior of the PA may result in the ACI. It can be noted that the ACI power is a nonlinear increasing function of the PA input power [90].

In the above context, authors in [90] recently studied the power allocation in CR networks by considering the nonlinear effects of the PA on the received SNR at the cognitive receiver and on the adjacent channel interference to the PRs. More specifically, the nonlinear effects of the power amplifier on the maximum achievable average SNR and also the secondary throughput have been studied by taking into account the effects of the resulting nonlinear ACIs to the PRs as constraints. It has been concluded that the power allocation scenario with average ACI constraints has less throughput degradation than the one with peak ACI constraints, and also attains higher maximum achievable average SNR. In the similar context, the recent contribution in [166] studies the effects of nonlinear PA on OFDM-based CR system from both in-band and out-of-band perspectives. Subsequently, a closed-form expression for the leakage power at adjacent channels has been derived. Further, an optimum power scaling factor for the input signal that maximizes the secondary rate has been derived by considering nonlinear effects on SINR under the constraints of the powers in the adjacent channels.

\section{B. Multicarrier Distortion}

For CR systems, NC-OFDM has been considered as an attractive physical layer technology due to its considerable higher spectral efficiency, immunity to the frequency selective fading channels, multipath delay spread tolerance, and high power efficiency [167]. In the conventional NC-OFDM based systems, the constituent subcarriers have to be turned off at the PUs channel to create spectrum notches in order to limit the interference perceived by the PUs [168]. Further, the guard band is required to be as narrow as possible in order to maximize the throughput of the NC-OFDM based CR system. Despite several advantages of the NC-OFDM based system, it mainly suffers from the following two drawbacks: (i) high PAPR of the transmitted NC-OFDM signals: Due to limited range of the High Power Amplifier (HPA), the NCOFDM signals with high PAPR become seriously clipped and nonlinear distortion is introduced, resulting in serious degradation of BER performance [91]. (ii) Large spectrum sidelobes: the larger spectrum sidelobes introduce interference to the adjacent PUs, resulting in the serious performance degradation of the adjacent PUs [92].

Several methods such as clipping, partial transmit sequence, active constellation extension, and tone reservation have been proposed in the literature in order to reduce the PAPR for the NC-OFDM based CR system (See references in [167]). However, these methods do not take into account of the side lobe suppression which is also a critical aspect for the NC-OFDM based system. For sidelobe suppression, many schemes such as extended active interference cancellation, pulse shaping, constellation adjustment, spectrum precoding, and sidelobe suppression with orthogonal projection have been proposed. Further, a novel signal cancellation scheme has been proposed in [167] for joint PAPR reduction and sidelobe suppression in the NC-OFDM based system. In the proposed joint scheme, a part of the outer constellation points on SU subcarriers have been dynamically extended while several signal cancellation symbols have been added to the PU subcarriers in order to generate the appropriate cancellation signal for joint PAPR reduction and sidelobe suppression.

\section{Synchronization Errors}

Frequency and phase offsets usually occur during the up conversion of the baseband signal to the passband and vice versa. In a practical CR transceiver, frequency offset may occur due to the result of carrier frequency mismatches of the Local Oscillators (LOs) in the transmitter and receiver sides, and the phase offset may occur due to phase jitter of the LOs and the phase mismatch between them [169]. Only a 
few works have been reported in the literature for analyzing the effect of frequency offset and phase noise on the detection performance of a CR [169]-[171]. Furthermore, IQ-mismatch error is usually generated when an amplitude imbalance or a quadrature error (phase difference is not exactly $90^{\circ}$ ) occurs between the I and Q branches. The IQ-mismatch generally causes an interference between the I and Q branches.

\section{Future RECOMMENDATIONS}

As discussed above, there are several imperfections in practical CR such as noise uncertainty, channel/interference uncertainty, noise/channel correlation, cyclic frequency mismatch, unknown (or imperfectly known) adjacent channel interferences, SNR/channel/DoA estimation errors, etc. Table IV provides the main available references that address these imperfections. Although the existing approaches attempt to examine some uncertainty issues, these approaches are still not robust against adjacent channel interferences, and cyclic frequency mismatch which may occur due to oscillator frequency offsets, Doppler shifts, or imperfect knowledge of the cyclic frequencies. Moreover, all these imperfections exist jointly and hence practical spectrum awareness algorithms may need to consider all of these aspects. Thus, further research may need to be performed to come up with a spectrum awareness mechanism by taking into account all these issues. One potential approach of addressing these uncertainties could be to examine the existing sensing methods while considering multiple antenna and cooperative sensing nodes. We highlight some of the open research issues related to $C R$ practical imperfections below.

\section{Calibration Error}

When there is no transmitted signal, the spectrum of practical received samples does not have a flat spectrum and incorporates several undesired spikes [172], [173]. This is mainly due to the non-flat transfer functions of the filter, IQ imbalance, spurious signal and phase noise which arise due to imperfections of oscillators, amplifiers, and limited dynamic ranges of the ADCs. Furthermore, when there is a primary (or other secondary) transmitted signal, such imperfections also arise at the transmitter side. Consequently, under the $H_{1}$ hypothesis, the effect of imperfections due to the transmitter and receiver further worsen the detection process.

To address the above issues, different calibration approaches can be applied. Calibration is an important step to be carried out at both the transmitter and receiver sides. However, in a CR network, the PTs and SRs are operated by different entities and performing calibration at both ends is not practical. For this reason, calibration is performed only at the cognitive receiver side. However, such a calibration is mainly device dependent. And in fact, if no calibration is performed, the existing algorithms discussed above may not ensure the desired awareness performance especially at a very low SNR region (i.e., $\leq-10 \mathrm{~dB}$ ) which is the desired region for CR application (see for example [99], [100]). Hence, the design of a general (and perhaps device independent) receiver calibration approach particularly for a CR is still an open research topic ${ }^{6}$. Along this side, it is also interesting to come up with a spectrum awareness mechanism/algorithm that do not assume an priori knowledge on the distribution of the noise samples, and is robust against undesired spikes and receiver calibration error.

\section{Quantization Errors}

Quantization is an important aspect of a CR transceiver and this process may result in two kinds of sources: (i) Quantization error, and (ii) Clipping error. An Automatic Gain Controller (AGC) is generally used to control the level of the input signal to the ADC. If the performance of the AGC is not optimized properly, it may result in the above errors, resulting in the detection performance degradation [93]. In this context, the contribution in [93] has analyzed the effect of quantization errors on the performance of the ED approach and serious degradation in the detection performance has been noted. The available literature related to this analysis is quite limited and it is crucial to investigate suitable measures in order to combat the effect of quantization errors while designing a practical $C R$ transceiver.

\section{Noise/Channel Correlation}

Although recent works [36], [140] have proposed new sensing bounds for SCN and ME based sensing under noise correlated scenarios, analyzing the effect of noise correlation on the performance of other detectors as well as investigation of new sensing bounds which provide improved detection performance under correlated scenarios is still an open research challenge. Future research should focus on investigating suitable approaches while considering these imperfections into account.

\section{SNR/Channel/DoA Estimation Errors}

In the existing power control based underlay techniques [71], the effect of SNR/channel estimation errors have not been taken into account. In practice, there may occur SNR/channel estimation errors due to inaccuracy of the estimation process and this may affect the performance of the power control-based underlay scheme. Furthermore, in most of the existing DoAbased cognitive beamforming literature, the effect of DoA estimation errors have been neglected [174], [175]. In practice, DoA estimation errors may cause the perturbations in the array response vector, leading to the need of robust cognitive beamforming techniques. In this context, it's an open research challenge to take the aforementioned errors into account while designing a practical underlay CR system.

\section{Limitations of Device Level and Cooperative SS}

The operation of the most of the current CRs rely on device level SS and decision-making. Such a process could be easily influenced by the environment, the capability and intention of the cognitive device. To this end, one important point is that such device level decision making about spectrum access is also problematic for the network operators, who may want to exert complete control over the spectrum. This might be one of the reasons why operators have been reluctant from using CRs.

Although cooperative SS has been investigated in various settings in order to address the aforementioned issue, the coop-

${ }^{6}$ Note that calibration can not eliminate the noise variance uncertainty. 


\begin{tabular}{l|l|l} 
Imperfection Type & Existing Methods (if any) & References \\
\hline Noise variance uncertainty & Cyclostationary based detector & {$[102]-[108],[110],[111]$} \\
& Autocorrelation based detector & {$[112]-[114]$} \\
& Covariance based detector & {$[115],[116]$} \\
& Eigenvalue based detector & {$[98],[120],[123],[125],[127]-[130]$} \\
& Moment based detector & {$[132]$} \\
& Max-Min SNR based detector & {$[135],[136]$} \\
Noise/channel correlation & Generalized energy detector (GED) & {$[99],[58],[139],[140]$} \\
Signal uncertainty & Eigenvalue based approach & {$[36],[5],[145]$} \\
Channel/interference uncertainty & Uncertainty models & {$[87],[140)$} \\
& Theoretical model based estimation & {$[150]$} \\
& Channel reciprocity & {$[151]$} \\
& Stochastic modeling & {$[152],[153]$} \\
CR transceiver imperfections & Worst case modeling & {$[154],[155]$} \\
Power amplifier nonlinearity & Reference distribution based approach & {$[149]$} \\
Multicarrier distortion & & {$[90],[166]$} \\
Synchronization errors & & {$[92],[167],[168]$} \\
Quantization errors & & {$[94],[95],[169]-[171]$} \\
\hline
\end{tabular}

erative burden (i.e., the requirement of the signalling links) and the induced delay are hindering its actual realization. Cooperative SS techniques, even with cyclostationary feature detection, require a large number of cooperating devices in order to reduce the probability of false alarms to acceptable levels. A large number of cooperating devices induces excessive latency, which could reduce the usefulness of SS. For example, by the time a decision is made, the spectrum availability and network conditions might already have changed. In this context, future research should focus on investigating suitable decision/data fusion schemes which can reduce the cooperation burden as well as the delay and at the same time can achieve the desired performance targets.

Furthermore, existing cooperative SS literature mostly considers homogeneous nodes considering identical node capabilities, equal number of antennas, identical received SNR, and equal sampling rate. However, in practice, the cooperating nodes are much likely to be heterogeneous in terms of the aforementioned aspects [176]. In this context, it's an important research challenge to investigate suitable cooperative schemes which can combine sensed information from heterogeneous nodes to make reliable decision with less cooperation overhead.

\section{Conclusions}

This paper has provided an overview of the existing CR approaches under practical imperfections. Several imperfections of a CR based system have been discussed and existing measures to counteract these imperfections have been reviewed. Although there has been a significant effort in proposing robust detectors against noise and channel uncertainty, other imperfections have still received less attention. The existing robust SS approaches against noise variance uncertainty have been detailed. Furthermore, existing approaches on channel/interference uncertainty, signal uncertainty, noise/channel correlation, CR transceiver imperfections have been described. Finally, some open issues which need to be considered carefully in the future research have been identified. It can be concluded that although several researchers have attempted to address the problems of practical imperfections, the available solutions are either applicable for specific scenarios or only mitigate the effect of one specific type of impairment. Therefore, it remains an open challenge to explore one common approach/framework which can combat the identified issues and is applicable for a wide range of scenarios.

\section{REFERENCES}

[1] J. Mitola and G. Q. Maguire, "Cognitive radio: making software radios more personal," IEEE Personal Commun., vol. 6, no. 4, pp. $13-18$, Aug. 1999.

[2] C.-S. Sum and et al, "Cognitive communication in TV white spaces: An overview of regulations, standards, and technology," IEEE Commun. Mag., vol. 51, no. 7, pp. 138-145, July 2013.

[3] S. K. Sharma, S. Chatzinotas, and B. Ottersten, "Satellite cognitive communications: Interference modeling and techniques selection," in Proc. 6th Advanced Satellite Multimedia Systems Conf. (ASMS) and 12th Signal Processing for Space Commun. Workshop (SPSC), Sept. 2012, pp. 111-118.

[4] S. K. Sharma, S. Chatzinotas, and B. Ottersten, "Cognitive radio techniques for satellite communication systems," in Proc. IEEE Veh. Technol. Conf. (VTC Fall), Sept. 2013, pp. 1-5.

[5] M. Nekovee, "Cognitive radio access to TV white spaces: Spectrum opportunities, commercial applications and remaining technology challenges," in Proc. IEEE Symp. on New Frontiers in Dynamic Spectrum Access Networks, Apr. 2010, pp. 1-10.

[6] S. Haykin, "Cognitive radio: brain-empowered wireless communications," IEEE J. Sel. Areas Commun., vol. 23, no. 2, pp. 201-220, Feb. 2005.

[7] A. Goldsmith, S. A. Jafar, I. Maric, and S. Srinivasa, "Breaking spectrum gridlock with cognitive radios: An information theoretic perspective," Proc. IEEE, vol. 97, no. 5, pp. 894 -914, May 2009.

[8] K. Patil, R. Prasad, and K. Skouby, "A survey of worldwide spectrum occupancy measurement campaigns for cognitive radio," in Proc. Int. Conf. Devices and Commun. (ICDeCom), Feb. 2011, pp. 1-5.

[9] E. Axell, G. Leus, E. G. Larsson, and H. V. Poor, "Spectrum sensing for cognitive radio: State-of-the-art and recent advances," IEEE Signal Process. Mag., vol. 29, no. 3, pp. 101-116, May 2012.

[10] T. Yucek and H. Arslan, "A survey of spectrum sensing algorithms for cognitive radio applications," IEEE Commun. Surveys \& Tutorials, vol. 11, no. 1, pp. 116-130, 2009.

[11] H. Sun, A. Nallanathan, C.-X. Wang, and Y. Chen, "Wideband spectrum sensing for cognitive radio networks: a survey," IEEE Wireless Commun., vol. 20, no. 2, pp. 74-81, Apr. 2013.

[12] I. F. Akyildiz, B. F. Lo, and R. Balakrishnan, "Cooperative spectrum sensing in cognitive radio networks: A survey," Physical Commun., vol. 4, no. 2011, pp. 40-62, 2011. 
[13] Y. Zeng, Y.-C. Liang, A. T. Hoang, and R. Zhang, "A Review on Spectrum Sensing for Cognitive Radio: Challenges and Solutions," EURASIP J. on Advances in Signal Process., vol. 2010, pp. 1-15, 2010.

[14] I. F. Akyildiz, W.-Y. Lee, M. C. Vuran, and S. Mohanty, "A survey on spectrum management in cognitive radio networks," IEEE Commun. Mag., vol. 46, no. 4, pp. 40-48, Apr. 2008.

[15] J. Wang, M. Ghosh, and K. Challapali, "Emerging cognitive radio applications: A survey," IEEE Commun. Mag., vol. 49, no. 3, pp. 7481, Mar. 2011

[16] M. T. Masonta, M. Mzyece, and N. Ntlatlapa, "Spectrum decision in cognitive radio networks: A survey," IEEE Commun. Surveys \& Tutorials, vol. 15, no. 3, pp. 1088-1107, 2013.

[17] E. Z. Tragos, S. Zeadally, A. G. Fragkiadakis, and V. A. Siris, "Spectrum Assignment in Cognitive Radio Networks: A Comprehensive Survey," IEEE Commun. Surveys \& Tutorials, vol. 15, no. 3, pp. 11081135, 2013.

[18] A. De Domenico, E. C. Strinati, and M. Di Benedetto, "A survey on MAC strategies for cognitive radio networks," IEEE Commun. Surveys \& Tutorials, vol. 14, no. 1, pp. 21-44, 2012.

[19] M. Naeem, A. Anpalagan, M. Jaseemuddin, and D. C. Lee, "Resource Allocation Techniques in Cooperative Cognitive Radio Networks," IEEE Commun. Surveys \& Tutorials, vol. 16, no. 2, pp. 729-744, 2014.

[20] A. Ahmad, S. Ahmad, M. H. Rehmani, N. U. Hassan, "A Survey on Radio Resource Allocation in Cognitive Radio Sensor Networks," IEEE Commun. Surveys \& Tutorials, vol. 17, no. 2, pp. 888-917, 2015.

[21] M. Bkassiny, Yang Le, S. K. Jayaweera, "A Survey on MachineLearning Techniques in Cognitive Radios," IEEE Commun. Surveys \& Tutorials, vol. 15, no. 3, pp. 1136-1159, 2013.

[22] B. Wang and K. J. R. Liu, "Advances in cognitive radio networks: A survey," IEEE J. Sel. Topics in Signal Process., vol. 5, no. 1, pp. 5-23, Feb. 2011.

[23] J. Palicot, C. Moy, and R. Hachemani, "Multilayer sensors for the sensorial radio bubble," Physical Commun., vol. 2, no. 1-2, pp. 151165, Sep. 2009.

[24] S. K. Sharma, "Interweave/Underlay Cognitive Radio Techniques and Applications in Satellite Communication Systems", Ph.D. dissertation, University of Luxembourg, Luxembourg, 2014, http://orbilu. uni.lu/handle/10993/18973.

[25] J. Riihijarvi, P. Mahonen, M. Petrova, and V. Kolar, "Enhancing cognitive radios with spatial statistics: From radio environment maps to topology engine," in Proc. 4th Int. Conf. on Cognitive Radio Oriented Wireless Networks and Commun., June 2009, pp. $1-6$.

[26] Y. Zhao, L. Morales, J. Gaeddert, K.K. Bae, J.-S. Um, and J. H. Reed, "Applying radio environment maps to cognitive wireless regional area networks," in Proc. 2nd IEEE Int. Symp. New Frontiers in Dynamic Spectrum Access Networks, Apr. 2007, pp. 115 -118.

[27] J.-W. Zhang, Q. Zhao, and J.-y. Zou, "The IEEE 802.22 WRAN system based on Radio Environment Map (REM)," in Proc. First Int. Workshop on Education Technol. and Computer Science, Mar. 2009, vol. 1, pp. $98-101$.

[28] S. Kawade and M. Nekovee, "Wireless options for high data-rate indoor users: Cognitive access to TV white space," in Proc. First UK-India Int. Workshop on Cognitive Wireless Systems, Apr. 2011.

[29] S. Subramani and et al, "Towards practical REM-based radio resource management," in Proc. Future Network Mobile Summit (FutureNetw), 2011, June 2011, pp. 1-8.

[30] J. Riihijarvi, P. Mahonen, and S. Sajjad, "Influence of transmitter configurations on spatial statistics of radio environment maps," in Proc. 20th Int. Symp. PIMRC, Sept. 2009, pp. 853-857.

[31] L. Bolea, J. Perez-Romero, R. Agusti, and O. Sallent, "Context discovery mechanisms for cognitive radio," in Proc. IEEE 73rd VTC (Spring), May 2011, pp. 1-5.

[32] M. M. Buddhikot and et al, "DIMSUMnet: new directions in wireless networking using coordinated dynamic spectrum," in Proc. 6th IEEE Int. Symp. World of Wireless Mobile and Multimedia Networks (WoWMoM), June 2005, pp. 78-85.

[33] B. Ramkumar, "Automatic modulation classification for cognitive radios using cyclic feature detection," IEEE Circuits and Systems Mag., vol. 9, no. 2, pp. 27-45, Second 2009 .

[34] A. Tsakmalis, S. Chatzinotas, and B. Ottersten, "Automatic modulation classification for adaptive power control in cognitive satellite communications," in Proc. 7th Advanced Satellite Multimedia Systems Conf. (ASMS) and the 13th Signal Process. for Space Commun. Workshop (SPSC), Sept. 2014, pp. 234-240.

[35] S. Chatzinotas, S. K. Sharma, and B. Ottersten, "Asymptotic analysis of eigenvalue-based blind spectrum sensing techniques," in Proc. IEEE
Int. Conf. Acoustics, Speech and Signal Process. (ICASSP), May 2013, pp. 4464-4468.

[36] S. K. Sharma, S. Chatzinotas, and B. Ottersten, "Eigenvalue based sensing and SNR estimation for cognitive radio in presence of noise correlation," IEEE Trans. Veh. Technol., vol. 62, no. 8, pp. 1-14, Sept. 2013.

[37] Z. Khalaf, A. Nafkha, and J. Palicot, "Blind spectrum detector for cognitive radio using compressed sensing and symmetry property of the second order cyclic autocorrelation," in Proc. 7th Int. Conf. CROWNCOM, June 2012, pp. 291-296.

[38] H. Wang, W. Jouini, R. Hachemani, J. Palicot, L. S. Cardoso, and M. Debbah, "Blind bandwidth shape recognition for standard identification using USRP platforms and sdr4all tools," in Proc. 6th Advanced Int. Conf. Telecommun., May 2010, pp. 147-152.

[39] F. A. Bhatti, G. B. Rowe, and K. W. Sowerby, "Spectrum sensing using feature vectors," in Proc. IEEE Int. Conf. Commun. Systems, Nov. 2012, pp. 448-452.

[40] H. Chen X. Chen and W. Meng, "Cooperative communications for cognitive radio networks-from theory to applications," IEEE Comm. Surveys \& Tutorials, vol. 16 , no. 3, pp. 1180-1192, 2014.

[41] Z. Quan, S. Cui, H. V. Poor, and A. H. Sayed, "Collaborative wideband sensing for cognitive radios," IEEE Signal Process. Mag., vol. 25, no. 6, pp. 60-73, Nov. 2008

[42] Z. Tian and G. B. Giannakis, "Compressed sensing for wideband cognitive radios," in Proc. IEEE Int. Conf. Acoustics, Speech and Signal Process., Apr. 2007, vol. 4, pp. 1357-1360.

[43] Z. Tian and G. B. Giannakis, "A wavelet approach to wideband spectrum sensing for cognitive radios," in Proc. Int. Conf. Cognitive Radio Oriented Wireless Networks and Commun., June 2006, pp. 1-5.

[44] Z. Quan, S. Cui, A. H. Sayed, and H. V. Poor, "Optimal multiband joint detection for spectrum sensing in cognitive radio networks," IEEE Trans. Signal Process., vol. 57, no. 3, pp. 1128-1140, Mar. 2009.

[45] Z. Quan, S. Cui, A. H. Sayed, and H. V. Poor, "Spatial-spectral joint detection for wideband spectrum sensing in cognitive radio networks," in Proc. IEEE ICASSP, Mar. 2008, pp. 2793-2796.

[46] M. A. Davenport, J. N. Laska, J. Treichler, and R. G. Baraniuk, "The pros and cons of compressive sensing for wideband signal acquisition: Noise folding versus dynamic range," IEEE Trans. Signal Process., vol. 60, no. 9, pp. 4628-4642, Sept. 2012.

[47] J. Meng, and et al, "Collaborative spectrum sensing from sparse observations in cognitive radio networks," IEEE J. Sel. Areas in Commun., vol. 29, no. 2, pp. 327-337, Feb. 2011.

[48] M. A. Davenport, P. T. Boufounos, M. B. Wakin, and R. G. Baraniuk, "Signal processing with compressive measurements," IEEE J. Sel. Topics in Signal Process., vol. 4, no. 2, pp. 445-460, Apr. 2010.

[49] M. Filo, A. Hossain, A. R. Biswas, and R. Piesiewicz, "Cognitive pilot channel: Enabler for radio systems coexistence," in Proc. 2nd Int. Workshop CogART, May 2009, pp. 17-23.

[50] R. J. Haines, "Cognitive pilot channels for femto-cell deployment," in Proc. 7th Int. Symp. Wireless Commun. Systems (ISWCS), Sept. 2010, pp. 631-635.

[51] S. M. Kay, Fundamentals of Statistical Signal Processing: Detection Theory, vol. II, Prentice-Hall PTR, First edition, 1998.

[52] S. K. Sharma, S. Maleki, S. Chatzintoas, J. Grotz, and B. Ottersten, "Implementation Issues of Cognitive Radio techniques for Ka-band (17.7-19.7 GHz) SatComs,” in Proc. 7th ASMS/13th SPSC, Sept. 2014, pp. $241-248$

[53] S. K. Sharma, S. Chatzinotas, and B. Ottersten, "Cognitive beamhopping for spectral coexistence of multibeam satellites," in Int. J. Satellite Commun. and Networking, Mar. 2014.

[54] A. Ijaz, A. B. Awoseyila, and B. G. Evans, "Improved SNR estimation for BPSK and QPSK signals," Electronic Letters, vol. 45, no. 16, pp. 858-859, July 2009.

[55] D. R. Pauluzzi and N. C. Beaulieu, "A comparison of SNR estimation techniques for the AWGN channel," IEEE Trans. Commun., vol. 48, no. 10 , pp. 1681-1691, Oct. 2000

[56] M. Fujii and Y. Watanabe, "A study on SNR estimation for cognitive radio," in Proc. IEEE Int. Conf. Ultra-Wideband (ICUWB), Sept. 2012, pp. $11-15$.

[57] T. Tian, H. Iwai, and H. Sasaoka, "Pseudo BER based SNR estimation for energy detection scheme in cognitive radio," in Proc. IEEE 75th Veh. Technol. Conf. (VTC Spring), May 2012, pp. 1-5.

[58] S. K. Sharma, S. Chatzinotas, and B. Ottersten, "SNR estimation for multi-dimensional cognitive receiver under correlated channel/noise," IEEE Trans. Wireless Commun., vol. 12, no. 12, pp. 6392-6405, Dec. 2013. 
[59] S. K. Sharma, S. Chatzinotas, and B. Ottersten, "Transmit beamforming for spectral coexistence of satellite and terrestrial networks," in Proc. 8th Int. Conf. on Cognitive Radio Oriented Wireless Networks (CROWNCOM), July 2013, pp. 275-281.

[60] S. K. Sharma, S. Chatzinotas, and B. Ottersten, "Spatial filtering for underlay cognitive SatComs," in Personal Satellite Services, R. Dhaou and et al, Eds., vol. 123 of Lecture Notes of the Institute for Computer Sciences, Social Informatics and Telecommun. Engg., pp. 186-198. Springer International Publishing, 2013.

[61] Y. Wang, Z. Tian, and C. Feng, "Sparsity order estimation and its application in compressive spectrum sensing for cognitive radios," IEEE Trans. Wireless Commun., vol. 11, no. 6, pp. 2116-2125, June 2012.

[62] Jia Meng, Wotao Yin, Husheng Li, E. Houssain, and Zhu Han, "Collaborative spectrum sensing from sparse observations using matrix completion for cognitive radio networks," in Proc. IEEE Int. Conf. ICASSP, pp. 3114-3117, Mar. 2010.

[63] S. K. Sharma, S. Chatzinotas, and B. Ottersten, "Compressive sparsity order estimation for wideband cognitive radio receiver," IEEE Trans. Signal Process., 2014, vol. 62, no. 19, Oct. 2014.

[64] I. Rashad, I. Budiarjo, and H. Nikookar, "Efficient pilot pattern for OFDM-based cognitive radio channel estimation - part 1," in Proc. 14th IEEE Symp. Commun. and Veh. Technol. in the Benelux, Nov. 2007, pp. 1-5.

[65] H. Abeida, "Data-aided SNR estimation in time-variant Rayleigh fading channels," IEEE Trans. Signal Process., vol. 58, no. 11, pp. 5496-5507, Nov. 2010.

[66] O. A. Dobre, Q. Zhang, S. Rajan, and R. Inkol, "Second-order cyclostationarity of cyclically prefixed single carrier linear digital modulations with applications to signal recognition," in Proc. IEEE Global Telecommun. Conf., Nov. 2008, pp. 1-5.

[67] Q. Zhang, O. A. Dobre, S. Rajan, R. Inkol, and E. Serpedin, "Cyclostationarity approach for the recognition of cyclically prefixed single carrier signals in cognitive radio," in Proc. IEEE Int. Conf. Commun. (ICC), May 2010, pp. 1-6.

[68] R. P. Darshini and S. Vijayprasath, "Parameter estimation of second order cyclostationarity signals using BT-SCLD and VBT-SCLD techniques," in Proc. Int. Conf. Electronics and Commun. Systems (ICECS), Feb. 2014, pp. 1-5.

[69] M. Kim, P. Kimtho, and J.-i. Takada, "Performance enhancement of cyclostationarity detector by utilizing multiple cyclic frequencies of ofdm signals," in Proc. IEEE Symp. on New Frontiers in Dynamic Spectrum Access Networks, Apr. 2010, pp. 1-8.

[70] J. Palicot, C. Moy and M. Debbah, "Introduction to Cognitive Radio," in Radio Engineering: From Software radio to Cognitive Radio, J. Palicot, Ed. Wiley, 2011, Ch. 1.

[71] S. K. Sharma, S. Chatzinotas, and B. Ottersten, "A hybrid cognitive transceiver architecture: Sensing-throughput tradeoff," in Proc. 9th Int. Conf. Cognitive Radio Oriented Wireless Networks (CROWNCOM), June 2014, pp. 143-149.

[72] X. Jiang, K.-K. Wong, Y. Zhang, and D. Edwards, "On hybrid overlayunderlay dynamic spectrum access: Double-threshold energy detection and Markov model," IEEE Trans. Veh. Technol., vol. 62, no. 8, pp. 4078-4083, Apr. 2013

[73] S. K. Sharma, S. Chatzinotas, and B. Ottersten, "Spectrum sensing in dual polarized fading channels for cognitive SatComs," in Proc. IEEE GLOBECOM, 2012, pp. 3419-3424.

[74] S. K. Sharma, S. Chatzinotas, and B. Ottersten, "Exploiting polarization for spectrum sensing in cognitive SatComs," in Proc. 7th Int. Conf. Cognitive Radio Oriented Wireless Networks (CROWNCOM), 2012, pp. $36-41$.

[75] S. K. Sharma, S. Chatzinotas, and B. Ottersten, "3D Beamforming for Spectral Coexistence of Satellite and Terrestrial Networks," in Proc. IEEE Veh. Technol. Conf. (to appear), Sept. 2015.

[76] A. Ghasemi, and E. S. Sousa, "Fundamental limits of spectrum-sharing in fading environments," IEEE Trans. Wireless Commun., vol. 6, no. 2, pp. 649 -658, Feb. 2007.

[77] FCC, "Establishment of an interference temperature metric to quantify and manage interference and to expand available unlicensed operation in certain fixed, mobile and satellite frequency bands," ET Docket 0.-289, 2003.

[78] T. W. Ban, W. Choi, B. C. Jung, and D. K. Sung, "Multi-user diversity in a spectrum sharing system," IEEE Trans. Wireless Commun., vol. 8 , no. 1 , pp. $102-106$, Jan. 2009.

[79] S. K. Sharma, S. Chatzinotas, and B. Ottersten, "Interference alignment for spectral coexistence of heterogeneous networks," EURASIP J. Wireless Commun. and Networking, vol. 46, Feb. 2013
[80] M. Costa, "Writing on dirty paper (corresp.)," IEEE Trans. Inform. Theory, vol. 29, no. 3, pp. 439 - 441, May 1983.

[81] S. I. Gel'fand and M. S. Pinsker, "Probl. Contr. and Inform. Theory," Coding for channel with random parameters, vol. 9, no. 1, pp. 19-31, 1980.

[82] S. Senthuran, A. Anpalagan, and O. Das, "Throughput analysis of opportunistic access strategies in hybrid underlay and overlay cognitive radio networks," IEEE Trans. Wireless Commun., vol. 11, no. 6, pp. 2024-2035, 2012.

[83] R. Tandra and A Sahai, "Fundamental limits on detection in low SNR under noise uncertainty," in Proc. Int. Conf. on Wireless Networks, Commun. and Mobile Computing, June 2005, vol. 1, pp. 464-469.

[84] R. Tandra and A. Sahai, "SNR walls for signal detection," IEEE J. Sel. Topics in Signal Process., vol. 2, no. 1, pp. 4-17, Feb. 2008.

[85] R. Tandra and A. Sahai, "Noise calibration, delay coherence and SNR walls for signal detection," in Proc. 3rd IEEE Symp. on DySPAN, Oct. 2008, pp. 1-11.

[86] A. Kaushik, S. K. Sharma, S. Chatzinotas, B. Ottersten, and F. K. Jondral, "Sensing-Throughput Tradeoff for Cognitive Radio Systems with Unknown Received Power," in Proc. 10th Int. Conf. Cognitive Radio Oriented Wireless Networks, Apr. 2015.

[87] M. Lopez-Benitez and F. Casadevall, "Signal uncertainty in spectrum sensing for cognitive radio," IEEE Trans. Commun., vol. 61, no. 4, pp. 1231-1241, Apr. 2013.

[88] D.-S. Shiu, G. J. Foschini, M. J. Gans, and J. M. Kahn, "Fading correlation and its effect on the capacity of multielement antenna systems," IEEE Trans. Commun., vol. 48, no. 3, pp. 502 -513, Mar. 2000.

[89] H. T. Hui, "Influence of antenna characteristics on MIMO systems with compact monopole arrays," IEEE Antennas and Wireless Propag. Letters, vol. 8, pp. $133-136,2009$.

[90] M. Majidi, A. Mohammadi, and A. Abdipour, "Analysis of the power amplifier nonlinearity on the power allocation in cognitive radio networks," IEEE Trans. Commun., vol. 62, no. 2, pp. 467-477, Feb. 2014.

[91] J.-C. Chen, and C.-P. Li, "Tone reservation using near-optimal peak reduction tone set selection algorithm for PAPR reduction in OFDM systems," IEEE Signal Process. Letters, vol. 17, no. 11, pp. 933-936, Nov. 2010.

[92] D. Qu, J. Ding, T. Jiang, and X. Sun, "Detection of non-contiguous OFDM symbols for cognitive radio systems without out-of-band spectrum synchronization," IEEE Trans. Wireless Commun., vol. 10, no. 2 , pp. 693-701, Feb. 2011

[93] S. M. Khadka and et al, "Practical detection issues of spectrum sensing for cognitive radio system," Tech. Rep., IEICE, 2009, Available online: http://www.ap.ide.titech.ac.jp/publications/Archive/.

[94] Y. Zeng and Y.-C. Liang, "Robustness of the cyclostationary detection to cyclic frequency mismatch," in Proc. IEEE Int. Symp. Personal Indoor and Mobile Radio Commun., Sept. 2010, pp. 2704-2709.

[95] B. Razavi, "Cognitive radio design challenges and techniques," IEEE J. Solid-State Circuits, vol. 45, no. 8, pp. 1542-1553, Aug. 2010.

[96] E. Ahmed, A. M. Eltawil, and A. Sabharwal, "Rate gain region and design tradeoffs for full-duplex wireless communications," IEEE Trans. Wireless Commun., vol. 12, no. 7, pp. 3556-3565, June 2013.

[97] M. Duarte, C. Dick, and A. Sabharwal, "Experiment-driven characterization of full-duplex wireless systems," IEEE Trans. Wireless Commun., vol. 11, no. 12, pp. 4296-4307, Nov. 2012.

[98] Y. Zeng and Y.-C. Liang, "Eigenvalue-based spectrum sensing algorithms for cognitive radio," IEEE Trans. Commun., vol. 57, no. 6, pp. 1784 -1793, June 2009.

[99] T. E. Bogale, L. Vandendorpe, and L. B. Le, "Wideband sensing and optimization for cognitive radio networks with noise variance uncertainty," IEEE Trans. Commun., No. 99, Jan. 2015.

[100] J. C. Merlano-Duncan, T. E. Bogale, and L. B. Le, "SDR Implementation of Spectrum Sensing for Wideband Cognitive Radio," in Proc. IEEE Veh. Technol. Conf. (VTC-Fall) (To appear), Sept. 2015.

[101] R. Tandra and A. Sahai, "SNR walls for signal detection," IEEE J. Sel. Topics Signal Process., vol. 2, no. 1, pp. 4 - 17, Feb. 2008.

[102] W. Gardner, "Spectral correlation of modulated signals: Part I-analog modulation," IEEE Trans. Commun., vol. 35, no. 6, pp. 584 - 594, June 1987

[103] W. Gardner, "Spectral correlation of modulated signals: Part II-digital modulation," IEEE Trans. Commun., vol. 35, no. 6, pp. 595 - 601, June 1987

[104] A. Tani and R. Fantacci, "A low-complexity cyclostationary based spectrum sensing for UWB and WiMAX coexistence with noise uncertainty," IEEE Trans. Veh. Techol., pp. 2940 - 2950, 2010. 
[105] T. E. Bogale and L. Vandendorpe, "Multi-cycle cyclostationary based spectrum sensing algorithm for OFDM signals with noise uncertainty in cognitive radio networks," in Proc. IEEE Military Commun. Conf. (MILCOM), Orlando, FL, USA, 29 Oct. - 01 Nov. 2012, pp. 1 - 6.

[106] W. Zeng and G. Bi, "Robust detection of OFDM signals for cognitive UWB in low SNR with noise uncertainty," in IEEE Personal, Indoor and Mobile Radio Commun. (PIMRC), Sep. 2008, pp. 1 - 5.

[107] J. Lunden, S. A. Kassam, and V. Koivunen, "Robust nonparametric cyclic correlation-based spectrum sensing for cognitive radio," IEEE Trans. Signal Process., vol. 58, no. 1, pp. 38 - 52, Jan. 2010.

[108] Z. Khalaf, A. Nafkha, and J. Palicot, "Blind spectrum detector for cognitive radio using compressed sensing," in Proc. IEEE Global Commun. Conf. (GLOBECOM), Dec. 2011.

[109] Z. Khalaf, A. Nafkha, and J. Palicot, "Blind spectrum detector for cognitive radio using compressed sensing and symmetry property of the second order cyclic autocorrelation," in Proc. IEEE Int. ICST Conf. Cognitive Radio Oriented Wireless Networks and Commun. (CROWNCOM), June 2012.

[110] B. Aziz and A. Nafkha, "Implementation of blind cyclostationary feature detector for cognitive radios using USRP," in Proc. IEEE 21st Int. Conf. Telecommun. (ICT), 2014, pp. $42-46$.

[111] M. Bkassiny, S. K. Jayaweera, Y. Li, and K. A. Avery, "Blind cyclostationary feature detection based spectrum sensing for autonomous self-learning cognitive radios," in Proc. IEEE Int. Conf. Commun. (ICC), 2012, pp. 1507 - 1511

[112] R. K. Sharma and J. W. Wallace, "Improved spectrum sensing by utilizing signal autocorrelation," in Proc. IEEE 69th Veh. Technol. Conf., Apr. 2009, pp. 1-5

[113] R. K. Sharma and J. W. Wallace, "Improved autocorrelation-based sensing using correlation distribution information," in Proc. Int. ITG Workshop on Smart Antennas (WSA), Feb. 2010, pp. 335-341.

[114] M. Naraghi-Pour and T. Ikuma, "Autocorrelation-based spectrum sensing for cognitive radios," IEEE Trans. Veh. Technol., vol. 59, no. 2, pp. 718-733, Feb. 2010.

[115] Y. Zeng and Y.-C. Liang, "Spectrum-sensing algorithms for cognitive radio based on statistical covariances," IEEE Trans. Veh. Technol., vol. 58, no. 4, pp. 1804-1815, May 2009.

[116] M. Jin, Y. Li, and H.-G. Ryu, "On the performance of covariance based spectrum sensing for cognitive radio," IEEE Trans. Signal Process., vol. 60, no. 7, pp. 3670-3682, July 2012.

[117] Y. Zeng, C. L. Koh, and Y.-C. Liang, "Maximum eigenvalue detection: Theory and application," in Proc. IEEE Int. Conf. Commun., May 2008, pp. $4160-4164$.

[118] P. Wang, and et al, "Multiantenna-assisted spectrum sensing for cognitive radio," IEEE Trans. Veh. Technol., vol. 59, no. 4, pp. 1791 -1800 , May 2010.

[119] L. S. Cardoso, M. Debbah, P. Bianchi, and J. Najim, "Cooperative spectrum sensing using random matrix theory," in Proc. 3rd Int. Symp. Wireless Pervasive Computing, May 2008, pp. 334 -338.

[120] F. Penna, R. Garello, and M. Spirito, "Cooperative spectrum sensing based on the limiting eigenvalue ratio distribution in Wishart matrices," IEEE Commun. Letters, vol. 13, no. 7, pp. 507 -509, July 2009

[121] A. Taherpour, M. Nasiri-Kenari, and S. Gazor, "Multiple antenna spectrum sensing in cognitive radios," IEEE Trans. Wireless Commun., vol. 9, no. 2, pp. 814-823, Feb. 2010.

[122] K. Hassan, R. Gautier, I. Dayoub, E. Radoi, and M. Berbineau, "Predicted eigenvalue threshold based spectrum sensing with correlated multiple-antennas," in Proc. IEEE 75th VTC Spring, May 2012, pp. 1 $-5$

[123] W. Zhang, G. Abreu, M. Inamori, and Y. Sanada, "Spectrum sensing algorithms via finite random matrices," IEEE Trans. Commun., vol. 60, no. 1, pp. $164-175$, Jan. 2012.

[124] A. Kortun, T. Ratnarajah, M. Sellathurai, Caijun Z., and C. B. Papadias, "On the performance of eigenvalue-based cooperative spectrum sensing for cognitive radio," IEEE J. Sel. Topics Signal Process., vol. 5, no. 1, pp. $49-55$, Feb. 2011

[125] L. Wei and O. Tirkkonen, "Spectrum sensing in the presence of multiple primary users," IEEE Trans. Commun., vol. 60, no. 5, pp. 1268-1277, May 2012.

[126] L. Wei and O. Tirkkonen, "Cooperative spectrum sensing of OFDM signals using largest eigenvalue distributions," in Proc. IEEE 20th Int. Symp. on Personal, Indoor and Mobile Radio Commun., Sept. 2009, pp. $2295-2299$

[127] P. Bianchi, M. Debbah, M. Maida, and J. Najim, "Performance of statistical tests for single-source detection using random matrix theory," IEEE Trans. Inform. Theory, vol. 57, no. 4, pp. 2400 -2419, Apr. 2011.
[128] L. Wei and O. Tirkkonen, "Analysis of scaled largest eigenvalue based detection for spectrum sensing," in Proc. IEEE Int. Conf. Commun., June 2011, pp. 1-5.

[129] R. Zhang, T. Lim, Y.-C. Liang, and Y. Zeng, "Multi-antenna based spectrum sensing for cognitive radios: A GLRT approach," IEEE Trans. Commun., vol. 58, no. 1, pp. 84 -88, Jan. 2010.

[130] R. Lopez-Valcarce, G. Vazquez-Vilar, and J. Sala, "Multiantenna spectrum sensing for cognitive radio: overcoming noise uncertainty," in Proc. 2nd Int. Workshop Cognitive Inform. Process., June 2010, pp. $310-315$.

[131] D. B. Williams, and D. H. Johnson, "Using the sphericity test for source detection with narrow-band passive arrays," IEEE Trans. Acoustics, Speech and Signal Process., vol. 38, no. 11, pp. $2008-2014$, Nov. 1990.

[132] T. E. Bogale, and L. Vandendorpe, "Moment based spectrum sensing algorithm for cognitive radio networks with noise variance uncertainty," in Proc. IEEE Conf. Inform. Sciences and Systems (CISS), Baltimore, MD, USA, Mar. 2013, pp. 1 - 5.

[133] M. R. Morelande and A. M. Zoubir, "Detection of phase modulated signals in additive noise," IEEE Signal Process. Letters, vol. 8, no. 7, pp. $199-202$, July 2001.

[134] A. Morello, and V. Mignone, "DVB-S2: The second generation standard for satellite broad-band services," IEEE Proc., vol. 94, no. 1, pp. $210-227,2006$

[135] T. E. Bogale, and L. Vandendorpe, "Max-Min SNR signal energy based spectrum sensing algorithms for cognitive radio networks with noise variance uncertainty," IEEE Trans. Wireless. Commun., vol. 30, no. 1, pp. $280-290$, Jan. 2014.

[136] T. E. Bogale, L. Vandendorpe, and L. B. Le, "Sensing throughput tradeoff for cognitive radio networks with noise variance uncertainty," in IEEE Conf. Cognitive Radio Oriented Wireless Networks (CROWNCOM), Oulu, Finland, 2 - 4 June 2014, pp. 435-441.

[137] Y. Zeng and Y.-C. Liang, "Eigenvalue-based spectrum sensing algorithms for cognitive radio," IEEE Trans. Commun., vol. 57, no. 6, pp. 1784 - 1793, June 2009.

[138] W. Ejaz, N. U. Hasan, and H. S. Kim, "SNR-based adaptive spectrum sensing for cognitive radio networks," Int. J. Innovative Computing, Inform. and Control, vol. 8, no. 9, pp. 6095-6105, 2012

[139] S. K. Sharma, S. Chatzinotas, and B. Ottersten, "The effect of noise correlation on fractional sampling based spectrum sensing," in Proc. IEEE Int. Commun. Conf., June 2013, pp. 2589-2594.

[140] S. K. Sharma, S. Chatzinotas, and B. Ottersten, "Maximum eigenvalue detection for spectrum sensing under correlated noise," in Proc. IEEE ICASSP, May 2014, pp. 4464-4468.

[141] S. Kim, J. Lee, H. Wang, and D. Hong, "Sensing performance of energy detector with correlated multiple antennas," IEEE Signal Process. Letters, vol. 16, no. 8, pp. 671 -674, Aug. 2009.

[142] V. R. S. Banjade, N. Rajatheva, and C. Tellambura, "Performance analysis of energy detection with multiple correlated antenna cognitive radio in Nakagami-m fading," IEEE Commun. Letters, vol. 16, no. 4, pp. $502-505$, Apr. 2012.

[143] L. P. Luo, P. Zhang, G. C. Zhang, and J. Y. Qin, "Spectrum sensing for cognitive radio networks with correlated multiple antennas," Electronics Letters, vol. 47, no. 23, pp. 1297 -1298, Nov. 2011.

[144] S. K. Sharma, S. Chatzinotas, and B. Ottersten, "Compressive SNR estimation for wideband cognitive radio under correlated scenarios," in IEEE Wireless Commun. and Networking Conf. (WCNC), Apr. 2014, pp. 713-718.

[145] S. Gong, P. Wang, W. Liu, and W. Zhuang, "Performance bounds of energy detection with signal uncertainty in cognitive radio networks," in Proc. IEEE INFOCOM, Apr. 2013, pp. 2238-2246.

[146] A. Ghasemi and E. S. Sousa, "Interference aggregation in spectrumsensing cognitive wireless networks," IEEE J. Sel. Topics Signal Process., vol. 2, no. 1, pp. 41-56, Feb. 2008.

[147] R. Couillet and M. Debbah, Random Matrix Methods for Wireless Communications, 1st ed. Cambridge University Press, 2011.

[148] A. Kaushik, S. K. Sharma, S. Chatzinotas, B. Ottersten, and F. K. Jondral, "Estimation-Throughput tradeoff for underlay cognitive radio systems," in Proc. IEEE Int. Commun. Conf. (to appear), June 2015.

[149] S. Gong, P. Wang, Y. Liu, and W. Zhuang, "Robust power control with distribution uncertainty in cognitive radio networks," IEEE J. Sel. Areas Commun., vol. 31, no. 11, pp. 2397-2408, Nov. 2013.

[150] W. Ren, Q. Zhao, and A. Swami, "Power control in cognitive radio networks: how to cross a multi-lane highway," IEEE J. Sel. Areas Commun., vol. 27, no. 7, pp. 1283-1296, Sept. 2009. 
[151] S. Huang, X. Liu, and Z. Ding, "Decentralized cognitive radio control based on inference from primary link control information," IEEE J. Sel. Areas Commun., vol. 29, no. 2, pp. 394-406, Feb. 2011.

[152] G. Zheng, S. Ma, K.-K. Wong, and T.-S. Ng, "Robust beamforming in cognitive radio," IEEE Trans. Wireless Commun., vol. 9, no. 2, pp. $570-576$, Feb. 2010

[153] E. Dall'Anese, S.-J. Kim, G. B. Giannakis, and S. Pupolin, "Power control for cognitive radio networks under channel uncertainty," IEEE Trans. Wireless Commun., vol. 10, no. 10, pp. 3541-3551, Oct. 2011.

[154] G. Zheng, K.-K. Wong, and B. Ottersten, "Robust cognitive beamforming with bounded channel uncertainties," IEEE Trans. Signal Process., vol. 57, no. 12, pp. $4871-4881$, Dec. 2009.

[155] Y. Huang, Q. Li, W.-K. Ma, and S. Zhang, "Robust multicast beamforming for spectrum sharing-based cognitive radios," IEEE Trans. Signal Process., vol. 60, no. 1, pp. 527-533, Jan. 2012.

[156] H. Xiao, K. Yang, and X. Wang, "Robust power control under channel uncertainty for cognitive radios with sensing delays," IEEE Trans. Wireless Commun., vol.12, no.2, pp. 646-655, Feb. 2013.

[157] P. Setoodeh, and S. Haykin, "Robust transmit power control for cognitive radio," Proc. IEEE, vol.97, no.5, pp. 915-939, May 2009.

[158] G. E. Monahan, "A survey of partially observable Markov decision processes: theory, models, and algorithms," Management Science, vol. 28, no. 1, pp. 116, Jan. 1982.

[159] J. Unnikrishnan, and V. V. Veeravalli, "Algorithms for Dynamic Spectrum Access With Learning for Cognitive Radio,” IEEE Trans. Signal Process., vol. 58, no. 2, pp. 750-760, Feb. 2010.

[160] F. H. Panahi, and T. Ohtsuki, "Optimal channel-sensing policy based on Fuzzy Q-Learning process over cognitive radio systems," in Proc. Int. Conf. Commun. (ICC), June 2013, pp. 2677-2682.

[161] Y. Wang, Y. Xu, L. Shen, C. Xu, and Y. Cheng, "Two-dimensional POMDP-based opportunistic spectrum access in time-varying environment with fading channels," Journal of Commun. and Networks, vol. 16, no. 2, pp. 217-226, Apr. 2014.

[162] S.-M. Cai, Yi Gong, "Cognitive beamforming for throughput maximization with statistical cross channel state information," IEEE Commun. Letters, vol.18, no.11, pp. 2031-2034, Nov. 2014

[163] M. M. Alam, S. Bhattarai, H. Liang and S. Shetty, "Robust transmit beamforming against steering vector uncertainty in cognitive radio networks," in Proc. IEEE Conf. Computer Commun. Workshops (INFOCOM WKSHPS), 2014, pp. 700-705.

[164] S. Chaudhari, J. Lunden, and V. Koivunen, "BEP walls for collaborative spectrum sensing," in Proc. IEEE Int. Conf. Acoustics, Speech and Signal Process. (ICASSP), May 2011, pp. 2984-2987.

[165] S. Chaudhari, J. Lunden, V. Koivunen, and H. V. Poor, "Cooperative sensing with imperfect reporting channels: Hard decisions or soft decisions?," IEEE Transs Signal Process., vol. 60, no. 1, pp. 18-28, Jan. 2012

[166] M. Baghani, A. Mohammadi, M. Majidi, and M. Valkama, "Analysis and rate optimization of OFDM-based cognitive radio networks under power amplifier nonlinearity," IEEE Trans. Commun., vol. 62, no. 10, pp. 3410-3419, Oct. 2014

[167] C. Ni, T. Jiang, and W. Peng, "Joint PAPR reduction and sidelobe suppression using signal cancellation in NC-OFDM based cognitive radio systems," IEEE Trans. Veh. Technol., vol. PP, no. 99, pp. 1-9, 2014.

[168] D. Qu, Z. Wang, and T. Jiang, "Extended active interference cancellation for sidelobe suppression in cognitive radio OFDM systems with cyclic prefix," IEEE Trans. Veh. Technol., vol. 59, no. 4, pp. 16891695, May 2010

[169] Z. Kollar and P. Horvath, "Modulation schemes for cognitive radio in white spaces," Radio Engineering, vol. 19, no. 4, pp. 511-517, Dec. 2010.

[170] Y. F. Sharkasi, D. McLernon, and M. Ghogho, "Robust spectrum sensing in the presence of carrier frequency offset and phase noise for cognitive radio," in Proc. Wireless Telecommun. Symp. (WTS), 2012, Apr. 2012, pp. 1-5.

[171] I. Nevat, G. W. Peters, and J. Yuan, "Blind spectrum sensing in cognitive radio over fading channels and frequency offsets," in Proc. IEEE Wireless Commun. and Networking Conf. (WCNC), Apr. 2012, pp. 1039-1043.

[172] H. Kim, C. Cordeiro, K. Challapali, and K. G. Shin, "An experimental approach to spectrum sensing in cognitive radio networks with offtheshelf ieee 802.11 devices," in Proc. IEEE Workshop Cognitive Radio Networks, Jan. 2007, pp. 1154 - 1158.

[173] T. Bogale and L. Vandendorpe, "USRP implementation of Max-Min SNR signal energy based spectrum sensing algorithms for cognitive radio networks," in Proc. IEEE Int. Conf. Commun. (ICC), 10 - 14 June 2014, pp. $1478-1482$.

[174] S.-M. Cai, and Y. Gong, "Cognitive beamforming for multiple secondary data streams with individual SNR constraints," IEEE Trans. Signal Process., vol. 61, no. 17, pp. 4189-4198, Sept. 2013.

[175] G. Bartoli, R. Fantacci, D. Marabissi, and M. Pucci, "Angular interference suppression in cognitive LTE-A femtocells," in Proc. Int. Wireless Commun. and Mobile Computing Conf., pp. 979-984, Aug. 2014.

[176] S. K. Sharma, S. Chatzinotas, and B. Ottersten, "Cooperative spectrum sensing for heterogeneous sensor networks using multiple decision statistics," in Proc. 10th Int. Conf. Cognitive Radio Oriented Wireless Networks (CROWNCOM), Apr. 2015.

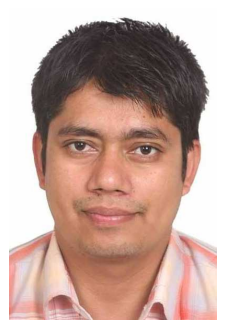

Shree Krishna Sharma (S'12-M'15) received the B.E. degree in electronics and communication from Birla Institute of Technology, Mesra, India, in 2004; the M.Sc. degree in information and communication engineering from the Institute of Engineering, Pulchowk, Nepal, in 2010; the M.A. degree in economics from Tribhuvan University Patan Multiple Campus, Patan, Nepal, and the M.Res. degree in computing science from Staffordshire University, Staffordshire, U.K., in 2011; and the Ph.D. degree in Computer Science from University of Luxembourg,

Luxembourg in 2014. Since November 2014, he has been working as a Postdoctoral Researcher in Interdisciplinary Centre for Security, Reliability and Trust, University of Luxembourg, Luxembourg.

In the past, he was also involved with Kathmandu University, Dhulikhel, Nepal, as a Teaching Assistant, and he served as a Part-Time Lecturer for eight engineering colleges in Nepal. He was with Nepal Telecom for more than four years as a Telecom Engineer in the field of information technology and telecommunication. Dr. Sharma has been involved in EU FP7 CoRaSat project, and EU H2020 SANSA, ESA project ASPIM, as well as Luxembourgish national projects Co2Sat, and SeMIGod. He received an Indian Embassy Scholarship for his B.E. study, an Erasmus Mundus Scholarship for his M.Res. study, an Aids Training-Research Ph.D. grant from the National Research Fund of Luxembourg, CROWNCOM 2015 Best Paper Award, and CSC best $\mathrm{PhD}$ thesis award (2013/2014). His research interests include cognitive wireless communications, resource allocation, and interference mitigation in heterogeneous wireless networks.

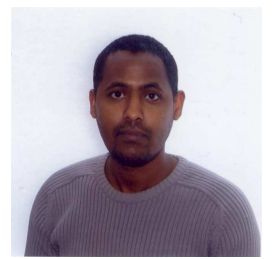

Tadilo Endeshaw Bogale (S'09-M'14) has received a BSc degree in Electrical Engineering from Jimma University, Jimma, Ethiopia. From 2004 to 2007, he was working in Ethio Telecom, Addis Ababa, Ethiopia. He received MSc and PhD degrees in Electrical Engineering from Karlstad University, Karlstad, Sweden and University Catholique de Louvain (UCL), Louvain la neuve, Belgium in 2008 and 2013, respectively. From January 2014 to October 2014, Tadilo was working as a postdoctoral researcher at the Institut National de la Recherche Scientifique (INRS), Montréal, Canada. Since November 2014, he has been working as a joint postdoctoral researcher with INRS and University of Western Ontario, London, Canada. Currently, he is working on assessing the potential technologies to enable the future 5G network. Specifically, his research focuses on the exploitation of massive MIMO and millimeter wave (mmWave) techniques for $5 \mathrm{G}$ network. His research interests include hybrid Analog-digital Beamforming for massive MIMO and mmWave systems, pilot contamination reduction for multicell massive MIMO systems, spectrum sensing and resource allocation for cognitive radio networks, robust (non-robust) transceiver design for multiuser MIMO systems, centralized and distributed algorithms, and convex optimization techniques for multiuser systems. He has recently organized a workshop on Cognitive Radio for 5G networks which was collocated in CROWNCOM 2015 conference. He was acting as a session chair for CISS, CROWNCOM and ICC conferences and NEWCOM workshop. Tadilo has also been serving as a TPC member on different international conferences such as CROWNCOM, PIMRC, ISWCS and VTC conferences. Tadilo is an IEEE member. 


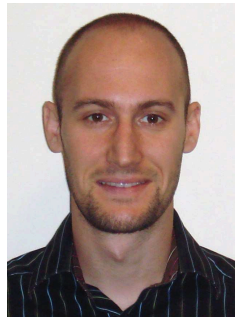

Symeon Chatzinotas (S'06-M'09-SM'13) received the M.Eng. in Telecommunications from Aristotle University of Thessaloniki, Greece and the M.Sc. and $\mathrm{Ph} . \mathrm{D}$. in Electronic Engineering from University of Surrey, UK in 2003, 2006 and 2009 respectively. He is currently a Research Scientist with the research group SIGCOM in the Interdisciplinary Centre for Security, Reliability and Trust, University of Luxembourg, managing $\mathrm{H} 2020$, ESA and FNR projects.

In the past, he has worked in numerous RD projects for the Institute of Informatics Telecommunications, National Center for Scientific Research Demokritos, the Institute of Telematics and Informatics, Center of Research and Technology Hellas and Mobile Communications Research Group, Center of Communication Systems Research, University of Surrey. He has authored more than 120 technical papers in refereed international journals, conferences and scientific books. His research interests are on multiuser information theory, cooperative/cognitive communications and wireless networks optimization. Dr Chatzinotas is the corecipient of the 2014 Distinguished Contributions to Satellite Communications Award, Satellite and Space Communications Technical Committee, IEEE Communications Society and CROWNCOM 2015 Best Paper Award. $\mathrm{He}$ is one of the editors of a book on "Cooperative and Cognitive Satellite Systems" published in 2015 by Elsevier and was involved in coorganizing the First International Workshop on Cognitive Radios and Networks for Spectrum Coexistence of Satellite and Terrestrial Systems (CogRaN-Sat) in conjunction with the IEEE ICC 2015, 8-12 June 2015, London, UK.

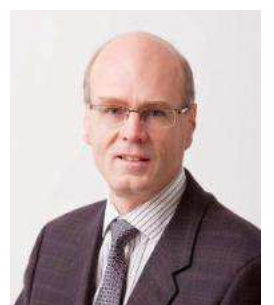

Björn Ottesten (S'87-M'89-SM'99-F'04) was born in Stockholm, Sweden, in 1961. He received the M.S. degree in electrical engineering and applied physics from Linköping University, Linköping, Sweden, in 1986 and the Ph.D. degree in electrical engineering from Stanford University, Stanford, CA, in 1989.

Dr. Ottersten has held research positions at the Department of Electrical Engineering, Linköping University, the Information Systems Laboratory, Stanford University, the Katholieke Universiteit Leuven, Leuven, and the University of Luxembourg. During 96/97, he was Director of Research at ArrayComm Inc, a start-up in San Jose, California based on Ottersten' s patented technology. He has co-authored journal papers that received the IEEE Signal Processing Society Best Paper Award in 1993, 2001, 2006, and 2013 and 3 IEEE conference papers receiving Best Paper Awards. In 1991, he was appointed Professor of Signal Processing at the Royal Institute of Technology (KTH), Stockholm. From 1992 to 2004, he was head of the department for Signals, Sensors, and Systems at KTH and from 2004 to 2008, he was dean of the School of Electrical Engineering at KTH. Currently, he is Director for the Interdisciplinary Centre for Security, Reliability and Trust at the University of Luxembourg. As Digital Champion of Luxembourg, he acts as an adviser to European Commissioner Neelie Kroes.

Dr. Ottersten has served as Associate Editor for the IEEE TRANSACTIONS ON SIGNAL PROCESSING and on the editorial board of IEEE Signal Processing Magazine. He is currently editor in chief of EURASIP Signal Processing Journal and a member of the editorial boards of EURASIP Journal of Applied Signal Processing and Foundations and Trends in Signal Processing. He is a Fellow of the IEEE and EURASIP and a member of the IEEE Signal Processing Society Board of Governors. In 2011, he received the IEEE Signal Processing Society Technical Achievement Award. He is a first recipient of the European Research Council advanced research grant. His research interests include security and trust, reliable wireless communications, and statistical signal processing.
Long Bao Le (S'04-M'07-SM'12) received the B.Eng. degree in electrical engineering from Ho Chi Minh City University of Technology, Vietnam, in 1999, the M.Eng. degree in telecommunications from Asian Institute of Technology, Thailand, in 2002, and the Ph.D. degree in electrical engineeringfrom the University of Manitoba, Canada, in 2007. He was a Postdoctoral Researcher at Massachusetts Institute of Technology (2008-2010) and University of Waterloo (2007-2008). Since 2010, he has been with the Institut National de la Recherche Scientifique (INRS), Université du Québec, Montréal, QC, Canada where he is currently an associate professor. His current research interests include smartgrids, cognitive radio, radio resource management, network control and optimization, and emerging enabling technologies for $5 \mathrm{G}$ wireless systems. He is a co-author of the book Radio Resource Management in Multi-Tier Cellular Wireless Networks (Wiley, 2013). Dr. Le is a member of the editorial board of IEEE TRANSACTIONS ON WIRELESS COMMUNICATIONS, IEEE COMMUNICATIONS SURVEYS AND TUTORIALS, and IEEE WIRELESS COMMUNICATIONS LETTERS. He has served as technical program committee co-chairs of the Wireless Access track at IEEE VTC2014-Fall, Wireless Networks track at IEEE VTC2011-Fall, and the Cognitive Radio and Spectrum Management track at IEEE PIMRC2011.

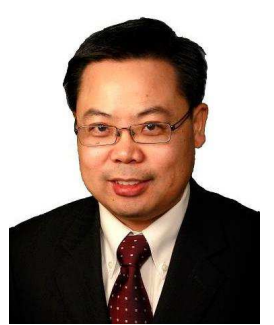

Xianbin Wang (S'98-M'99-SM'06) is a Professor and Canada Research Chair at Western University, Canada. He received his Ph.D. degree in electrical and computer engineering from National University of Singapore in 2001.

Prior to joining Western, he was with Communications Research Centre Canada as a Research Scientist/Senior Research Scientist between July 2002 and Dec. 2007. From Jan. 2001 to July 2002, he was a system designer at STMicroelectronics, where he was responsible for system design for DSL and Gigabit Ethernet chipsets. His current research interests include adaptive wireless systems, 5G networks, communications security, and distributed ICT systems. Dr. Wang has over 200 peer-reviewed journal and conference papers on various communication system design issues, in addition to 24 granted and pending patents and several standard contributions.

Dr. Wang was the recipient of three IEEE Best Paper Awards. He currently serves as an Associate Editor for IEEE Wireless Communications Letters, IEEE Transactions on Vehicular Technology and IEEE Transactions on Broadcasting. He was also an editor for IEEE Transactions on Wireless Communications between 2007 and 2011. Dr. Wang is a Senior Member of IEEE and an IEEE Distinguished Lecturer. He was involved in a number of IEEE conferences including GLOBECOM, ICC, WCNC, VTC, ICME and CWIT, in different roles such as symposium chair, tutorial instructor, track chair, session chair and TPC chair. 